Review Article

\title{
Prospects on Time-Domain Diffuse Optical Tomography Based on Time-Correlated Single Photon Counting for Small Animal Imaging
}

\author{
Yves Bérubé-Lauzière, ${ }^{1,2,3}$ Matteo Crotti, ${ }^{4}$ Simon Boucher, ${ }^{1}$ Seyedrohollah Ettehadi, ${ }^{1}$ \\ Julien Pichette, ${ }^{1}$ and Ivan Rech ${ }^{4}$ \\ ${ }^{1}$ Department of Electrical and Computer Engineering, Université de Sherbrooke, 2500 Boulevard de l'Université, \\ Sherbrooke, QC, Canada J1K 2R1 \\ ${ }^{2}$ Centre d'Imagerie Moléculaire de Sherbrooke (CIMS), Centre de Recherche du Centre Hospitalier Universitaire de Sherbrooke \\ (CR-CHUS), 3001 12e Avenue Nord, Sherbrooke, QC, Canada J1H 5N4 \\ ${ }^{3}$ Institut Interdisciplinaire d'Innovation Technologique (3IT), Parc Innovation, Pavillon P2, 3000 Boulevard de l'Université, \\ Sherbrooke, QC, Canada J1K 0A5 \\ ${ }^{4}$ Dipartimento di Elettronica, Informazione e Bioingegneria, Politecnico di Milano, Piazza Leonardo da Vinci 32, 20133 Milano, Italy
}

Correspondence should be addressed to Yves Bérubé-Lauzière; yves.berube-lauziere@usherbrooke.ca

Received 2 October 2015; Revised 5 December 2015; Accepted 20 December 2015

Academic Editor: Rickson C. Mesquita

Copyright (C) 2016 Yves Bérubé-Lauzière et al. This is an open access article distributed under the Creative Commons Attribution License, which permits unrestricted use, distribution, and reproduction in any medium, provided the original work is properly cited.

\begin{abstract}
This paper discusses instrumentation based on multiview parallel high temporal resolution $(<50 \mathrm{ps})$ time-domain (TD) measurements for diffuse optical tomography (DOT) and a prospective view on the steps to undertake as regards such instrumentation to make TD-DOT a viable technology for small animal molecular imaging. TD measurements provide information-richest data, and we briefly review the interaction of light with biological tissues to provide an understanding of this. This data richness is yet to be exploited to its full potential to increase the spatial resolution of DOT imaging and to allow probing, via the fluorescence lifetime, tissue biochemical parameters, and processes that are otherwise not accessible in fluorescence DOT. TD data acquisition time is, however, the main factor that currently compromises the viability of TD-DOT. Current high temporal resolution TD-DOT scanners simply do not integrate sufficient detection channels. Based on our past experience in developing TD-DOT instrumentation, we review and discuss promising technologies to overcome this difficulty. These are single photon avalanche diode (SPAD) detectors and fully parallel highly integrated electronics for time-correlated single photon counting (TCSPC). We present experimental results obtained with such technologies demonstrating the feasibility of next-generation multiview TD-DOT therewith.
\end{abstract}

\section{Introduction}

Similarly to widespread medical imaging modalities, such as X-ray computed tomography (CT), magnetic resonance imaging (MRI), nuclear imaging (positron emission tomography (PET) and single photon emission computed tomography (SPECT)), and ultrasound (US), diffuse optical tomography (DOT) seeks to obtain deep $(\geq 1 \mathrm{~cm})$ interior images of living organisms with noninvasive exterior-only measurements. Rather than using X-rays, radio waves and magnetic fields, gamma rays, or mechanical waves, DOT uses light and optical techniques (laser or incandescent sources, light detectors, and optical components) to achieve this. More precisely, DOT aims at reconstructing 3D spatial maps of the optical properties of biological tissues (absorption and scattering coefficients) in the case of intrinsic imaging or of the concentration of a light-emitting compound in the cases of fluorescence or bioluminescence imaging $[1,2]$. This is achieved by measuring light emerging from a tissue at several locations on its boundary and feeding the measured data to an image reconstruction algorithm that will recover an image therefrom. 
Optical methods present several interesting features for the purpose of medical imaging. First, light in the visible and near-infrared (NIR) ranges is nonionizing, and, thus, it does not harm biological tissues provided some maximum exposure limits are not exceeded [3,4]. Second, optical methods are sensitive to blood oxygenation owing to the fact that blood absorption, which is a major component of the optical absorption of tissues, is directly linked to the oxy- and deoxyhemoglobin concentrations in blood $[1,5]$ through the relationship

$$
\mu_{a, \text { blood }}(\lambda)=\ln (10)\left[\varepsilon_{\mathrm{HbO}_{2}}(\lambda) C_{\mathrm{HbO}_{2}}+\varepsilon_{\mathrm{Hb}}(\lambda) C_{\mathrm{Hb}}\right] .
$$

Here $\mu_{a \text {,blood }}$ is the optical absorption coefficient of blood, which is a function of wavelength $\lambda, \varepsilon_{\mathrm{HbO}_{2}}$ and $\varepsilon_{\mathrm{Hb}}$ are, respectively, the wavelength dependent molar extinction coefficients of oxyhemoglobin $\left(\mathrm{HbO}_{2}\right)$ and deoxyhemoglobin $(\mathrm{Hb})$, and $C_{\mathrm{HbO}_{2}}$ and $C_{\mathrm{Hb}}$ are their respective concentrations. Figure 1 depicts absorption spectra of $\mathrm{Hb}$ and $\mathrm{HbO}_{2}$ at typical blood concentrations $(150 \mathrm{mg} / \mathrm{mL})$ along with those of other important chromophores present in biological tissues (water, fat, and melanin). For water and fat, the absorption spectra are for pure water and pure fat. For melanin, the spectrum displayed is that typical of skin obtained with the formula $\mu_{a}\left(\mathrm{~cm}^{-1}\right)=1.70 \times 10^{12} \times \lambda^{-3.48}$, with $\lambda$ given in $\mathrm{nm}$. Blood oxygenation is not accessible with other medical imaging techniques, except for functional MRI (fMRI) through blood oxygen level dependent (BOLD) signals, which, however, represent a complex mixture of blood flow, blood volume, and oxygen metabolism [6]. There is no simple relationship for BOLD signals such as (1) to directly access oxy- and deoxyhemoglobin concentrations. Third, optical imaging proves to be of high interest for in vivo molecular imaging due to the wide availability and relative ease of synthesizing molecular fluorescent and bioluminescent probes that possess high specificity. In contrast, probes for PET require handling radioactivity and synthesizing molecules to which a large radioactive atom (e.g., copper) needs to be attached, which is chemically challenging [7]. Similarly, probes for MRI also require binding large and heavy atoms (typically gadolinium). Furthermore, owing to MRI's very low sensitivity, large probe concentrations are often necessary to obtain sufficient signal. This can pose problems related to toxicity or to the possibility of altering the biological system under study via pharmacological effects [8]. Finally, optical technology is relatively cheap compared to other medical imaging technologies.

DOT finds applications in diagnostic imaging of the breast [9-12], joints [13], and prostate [14, 15], as well as in brain imaging for diagnostic and fundamental research purposes [16-18], whereas fluorescence DOT (FDOT) and bioluminescence DOT (BDOT) find most of their applications in small animal preclinical imaging [2, 8, 19-24], whereby one seeks to study biomolecular processes in small animal models of human diseases using light-emitting probes. This is of interest in a wide range of fields: (i) biology and medicine to develop an understanding of the fundamental mechanisms of disease development; (ii) pharmacology to label a drug or medication and follow its progression to see

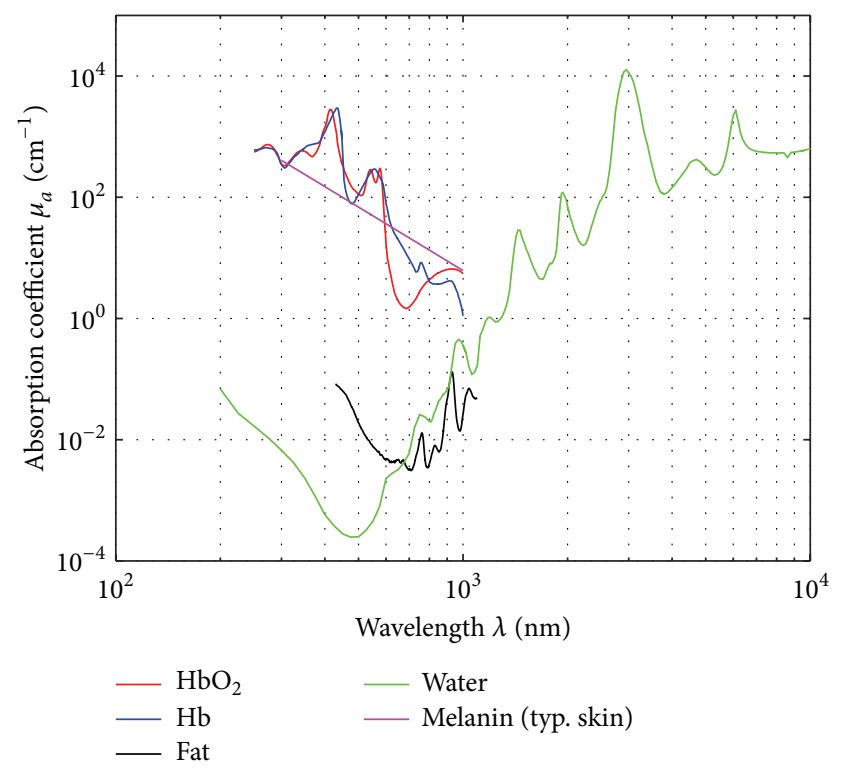

FIGURE 1: Absorption spectra of chromophores (absorbers) present in biological tissues. Data and equations for generating these plots were taken from Prahl and Jacques' WEB page [5]; see text for details.

if it reaches targeted tissues (this can lead to faster drug development, which is of great interest to the pharmaceutical industry), or to follow the evolution of a pathology under treatment on the same subject (longitudinal studies) to monitor treatment efficacy, which also allows reducing the number of animals used/sacrificed, a subject of great ethical interest; (iii) oncology for cancer cell tracking by labeling specific membrane proteins and monitor medication and radiotherapy effects [25]; (iv) toxicology to study chemicals and drugs toxicity and their effects on the health of living organisms and in several other areas ( $\mathrm{pH}$ imaging, antibody labeling, enzymatic reactions, peptides imaging, etc.).

DOT involves 3 major aspects: (1) how light interacts with biological tissues, which determines the imaged parameters and leads to light propagation modeling; (2) instrumentation to carry out multiview measurements and acquire the data necessary for tomographic imaging; and (3) tomographic image reconstruction algorithms that allow reconstructing interior images from the acquired data.

DOT is a relatively immature imaging modality compared to CT, MRI, PET, SPECT, and US, primarily because the image reconstruction problem is highly ill-posed and illconditioned owing to the high degree of light scattering in tissues. One way of reducing this ill-posedness in both DOT and FDOT is to resort to time-domain (TD) optical measurements (note: this excludes BDOT since bioluminescence emission is intrinsically a continuous-wave signal). This will be discussed further later on.

The TomOptUS group at Université de Sherbrooke has undertaken the development of a TD-DOT scanner for small animal (mouse) molecular imaging. The first generation of this scanner has been discussed at length in [26]. It is not the purpose to go over this again here in detail but rather to overview the architecture and hardware, summarize the highlights and shortcomings of that first generation along 


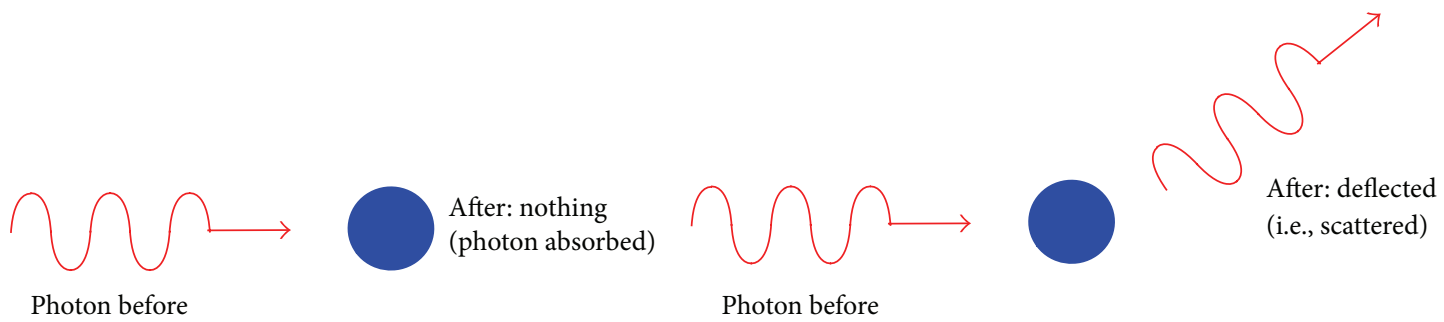

(a) Absorption

(b) Scattering

FIGURE 2: Microscopic view of (a) absorption of a photon by an absorber (represented by the blue circle) and (b) scattering of a photon by a scatterer (blue circle). See text for details.

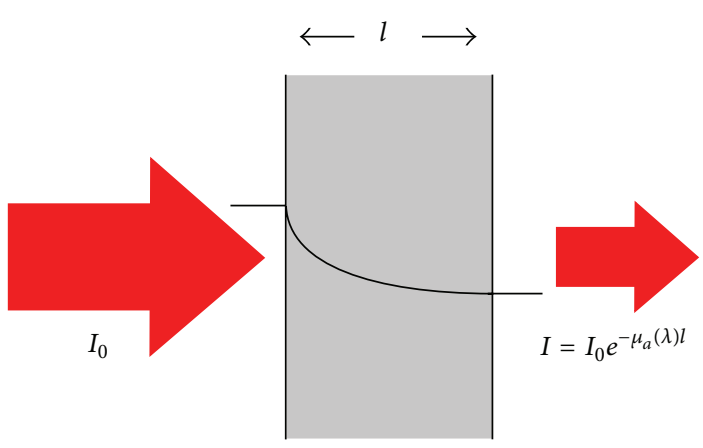

(a) Absorption

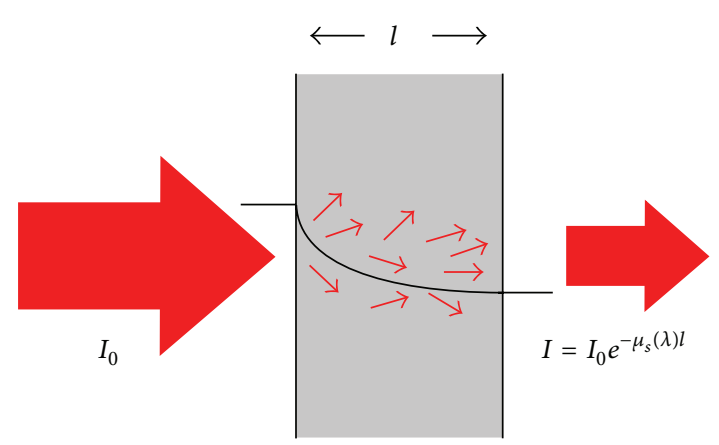

(b) Scattering

FIGURE 3: Macroscopic view of (a) absorption and (b) scattering.

with some illustrative results, and more importantly discuss what will come next and the work undertaken in that direction and associated early results.

Although image reconstruction will be alluded to, the present paper focusses on hardware aspects of TD-DOT and on some future prospects on it, notably as regards enabling technologies for the success of TD-DOT, specifically single photon avalanche diodes (SPADs) and parallel multichannel time-correlated single photon counting (TCSPC), two areas in which the SPADlab at Politecnico di Milano (PoliMi) has made significant contributions in recent years.

The paper is subdivided as follows. Section 2 reviews the basics of how light interacts with biological tissues. Hardware requirements for TD-DOT and associated challenges are discussed in Section 3. This is followed by Section 4 that overviews the first-generation TomOptUS scanner along with imaging results obtained with it. Section 5 discusses key advances related to TCSPC technology that will allow improving on the performances of the first-generation scanner; a second-generation preliminary prototype is presented along with associated imaging results which are compared with those of the first generation. Finally, Section 6 draws some conclusions, gives a summary, and presents an overview of current work.

\section{Review on the Interaction of Light with Biological Tissues}

When a photon propagates through a biological tissue, it can undergo two types of interactions: absorption and scattering.
Figure 2 illustrates simplistic microscopic views of these two processes, whereby a photon absorbed disappears as its energy is converted into some other form (typically heat) by an absorber (typically some complex molecule in biological tissues, such as hemoglobin), whereas scattering causes the photon to be deflected in another direction without loss of energy (elastic scattering is considered here; scatterers in biological tissues are generally structures of different sizes such as cells, cell nuclei, and mitochondria down to cell membranes [1]).

The probability for a photon coming from a direction specified by the unit vector $\widehat{\mathbf{s}}^{\prime}$ to be scattered in another direction $\widehat{\mathbf{s}}$ is given by the so-called phase function $p\left(\widehat{\mathbf{s}}^{\prime}, \widehat{\mathbf{s}}\right)$ which can be obtained from electromagnetic theory such as Mie's theory for scattering by spheres or ultimately from quantum mechanics by considering the fundamental processes underlying scattering (in tissue optics, however, one never goes that far as it is not necessary). In biomedical optics, the Henyey-Greenstein phase function, which has been determined empirically to represent well the scattering directional properties of biological tissues [27-29], is often resorted to $[1,30,31]$.

Absorption and scattering provide the two possible contrast mechanisms for optically imaging a tissue in its native state. On a macroscopic scale, absorption, characterized by the so-called absorption coefficient denoted $\mu_{a}$, is responsible for the attenuation of light energy in a medium (Figure 3(a)). The macroscopic intensity of a light beam is exponentially attenuated following Beer's law $I=I_{0} e^{-\mu_{a}(\lambda) l}$, where $I_{0}$ is the injected intensity and $I$ is the intensity after having traversed 


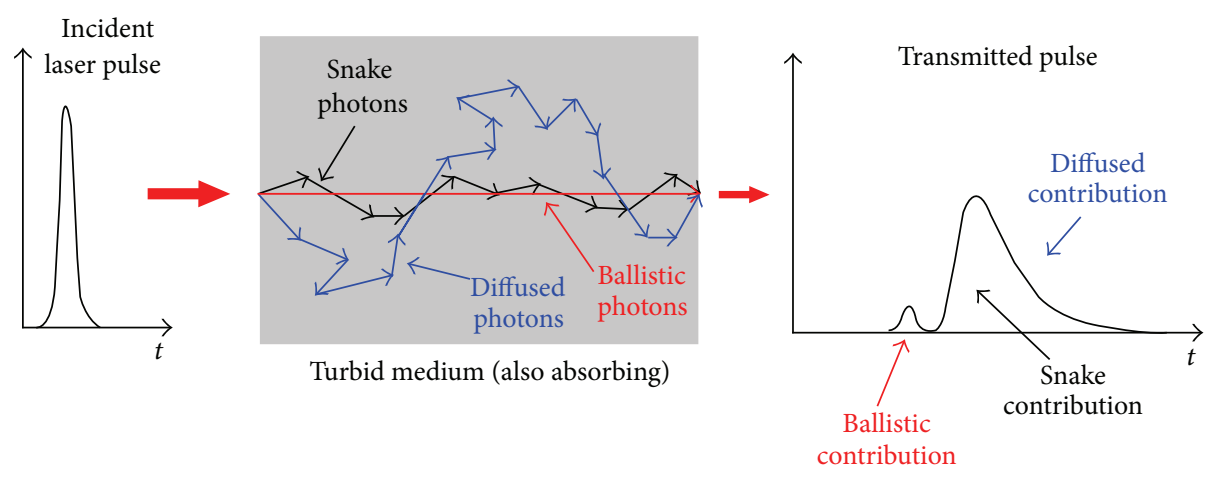

FIGURE 4: Transmitted pulse representing the photons time-of-flight distribution (TPSF) measured following injection of an ultrashort laser pulse into a turbid medium (scattering and absorbing).

the medium with thickness $l$. The absorption coefficient $\mu_{a}$ is wavelength dependent as indicated by the dependence on $\lambda$. Absorption in biological tissues is weakest in the near infrared (NIR) between 650 and $1000 \mathrm{~nm}$ owing to the absorption spectra of $\mathrm{HbO}_{2}$ and $\mathrm{Hb}$ (Figure 1), which are the main absorbing chromophores. Hence, light in that range (the so-called therapeutic window) is preferably used to image biological tissues as it can travel centimeters before being absorbed, whereas visible light is practically completely absorbed after a few millimeters. Scattering, which is strong in biological tissues, is responsible for the macroscopic diffusive nature of light propagation therein (much like heat flow in a metal), a fact that degrades the spatial resolution achievable by optical imaging. Macroscopically, scattering is characterized by the so-called scattering coefficient denoted by $\mu_{s}$ along with the phase function. For scattering, the macroscopic intensity also obeys Beer's law, with the scattering coefficient $\mu_{s}$ of the medium characterizing the strength at which scattering occurs within the medium (Figure 3(b)). Under some simplifications (diffusion theory), scattering is also customarily characterized by the reduced scattering coefficient $\mu_{s}^{\prime}=(1-g) \mu_{s}$, where $g$ is the anisotropy factor representing the average cosine of the angle between the incoming and scattered directions [1].

Photons in diffusive media can be thought of as propagating along 3 different regimes. These regimes can be discriminated by way of TD measurements made at the boundary of a medium of the photon time-of-flight curve [32] (the so-called time point-spread function (TPSF)Figure 4). First are ballistic photons, which by definition propagate in straight lines, only suffering absorption without being scattered (their contribution is grossly exaggerated in Figure 4). Such photons are, however, too few to be used for any practical purposes in tissue imaging as soon as the propagation distance exceeds a few millimeters $(\approx 2$ $3 \mathrm{~mm}$ ), beyond which practically all photons have suffered a scattering event [32]. Second are the so-called snake or early photons which suffer few scattering events and can be considered to propagate along nearly straight paths $[33,34]$. These are the photons appearing early in a measurement and contribute to the rising edge of a TPSF (Figure 4). Finally are diffused photons following highly meandering paths. These are of lesser interest for imaging as their paths are completely random, making the regions of the medium which they probe unknown. They are ultimately responsible for the blurry nature of DOT images.

Since TD measurements directly allow discriminating the different regimes of photon propagation, they are generally accepted as providing for the richest data $[1,2]$. Indeed, the shape of a TPSF directly depends on the absorption and scattering coefficients $\mu_{a}$ and $\mu_{s}$, whereas continuous-wave (CW) measurements provide only a number (light intensity) with no signature about how much a photon may have suffered scattering events while propagating. TD-DOT aims at exploiting this data richness to improve the quality of the reconstructed images in terms of obtaining higher spatial resolution and more quantitative imaging. This has been shown to be the case in both intrinsic and fluorescence imaging [3537], and indications of this can also be found in our work on the development of image reconstruction algorithms [38]. Further, TD measurements also allow probing parameters not accessible with CW measurements, such as the fluorescence lifetime, which is the mean time for an excited fluorescent molecule to relax to its ground state. In simplest form, this relaxation is described by a single decaying exponential. In tissue, this decay is convolved with TPSFs to account for the diffusive nature of light propagation resulting in a compound curve being measured and to be called here a fluorescence TPSF (FTPSF).

Lifetime is important for imaging. It is widely used in microscopy (fluorescence lifetime imaging (FLIM)) [39], because the lifetime of a fluorescent molecule is sensitive to the biochemical environment in tissues (e.g., the $\mathrm{pH}$ ) and to chemical binding. It thus offers a high potential for in vivo molecular imaging. Of particular interest is Förster resonance energy transfer (FRET) [40-42] whereby two fluorescent molecules, a donor and an acceptor interact, with the donor's emission spectrum overlap with the acceptor's absorption spectrum. As a donor gets in proximity to an acceptor, its fluorescence will get absorbed ("quenched") by the acceptor, with quenching getting more efficient the shorter the distance between the two. This leads to a modulation of the donors fluorescence lifetime that depends on the donor-acceptor distance. More precisely, the transfer rate of energy between 
the donor and the acceptor varies as the inverse of the 6th power of their distance. The effect of the increase of the transfer rate from the donor to the acceptor is to decrease the donors fluorescence lifetime as the donor has access to a faster channel to return back to its ground state. This effect can notably be used to study the conformation of molecules [43]. FRET has of course an influence on intensity-based measurements because if quenching is stronger, the light signal will be weaker. However, intensity-based measurements are also dependent on the fluorescent probes concentration, thus confounding quenching with concentration, which is not the case with lifetime measurements. Another application of FRET is in the study of interactions between biomolecules (e.g., protein-protein interactions or antibody labeling). With one type of biomolecule labeled with a donor and the other type with an acceptor, the donor's lifetime will be different depending on whether the molecules interact or not (when interacting, they will be a shorter distance apart). Of course not all molecules will interact, and thus the overall signal will be formed by two decaying exponentials. Fitting measured signals with a double-exponential model allows determining the lifetimes associated with the interacting and noninteracting molecules along with the fraction of interacting molecules, which is not possible with intensitybased measurements. As a note, FRET allows the design of smart probes that are normally in the OFF state (no light emission) but start emitting light (ON state) when cleaved by a specific enzyme [22]. In this case intensity-based measurements suffice.

\section{Challenges and Requirements in TD-DOT}

Typically, to carry out TD measurements, one shines an ultrashort excitation light pulse (ideally approximated by a Dirac delta function in time and typically output by an ultrafast laser) on the medium of interest and measures the pulse's temporal shape after its propagation through the medium. In effect, one measures the transfer function of the medium of which Figure 4 exemplifies a case. Such measurements can be performed with a certain number of means depending on the characteristics of the medium, which determines the amplitude and the time scale of the duration of the pulse to be measured. In biological tissues, an ultrashort pulse will typically temporally broaden by about 1 ns FWHM per centimeter of tissue traversed. For instance, laboratory mice, of interest in our work, typically have a diameter of about $2.5 \mathrm{~cm}$ and thus typical measured pulse durations are on the order of $2.5 \mathrm{~ns}$. To resolve such signals, which can be modeled as the convolution of the true signal from the process to be measured with the system's IRF, the system must have a very high temporal resolution on the order of $100 \mathrm{ps}$ or less in order not to distort (smear out) the signal too severely and lose information. Mathematically, the true signal can be recovered by deconvolving the IRF out of the measured signal, but since deconvolution is an ill-posed problem, it is advised to keep distortions to a minimum with deconvolution, then giving better results [44, 45]. Furthermore, an excitation pulse is attenuated by several orders of magnitude after its propagation through a biological

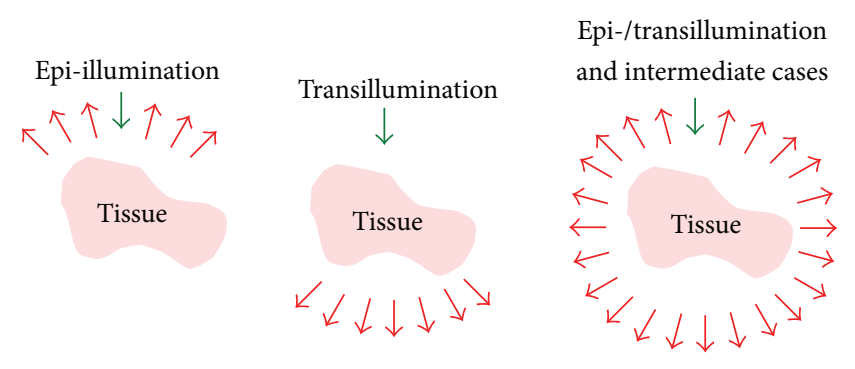

FIGURE 5: Multiview illumination and detection geometries.

tissue due to absorption and owing to its spreading in all directions as a consequence of scattering. Thus, imaging instrumentation shall be able to measure very faint optical signals (a few picowatts are typical). As regards fluorescence measurements, fluorophores used in optical imaging typically have fluorescence lifetimes ranging from 500 ps to $2-3 \mathrm{~ns}$. Again, resolving exponential decay curves with such lifetimes requires temporal resolutions down to 100 ps or less. In view of FRET measurements, discriminating nearby fluorescence lifetimes may call for even finer temporal resolutions. Fluorescence signals emanating from biological tissues also tend to be extremely faint. All these requirements (very short and weak signals) are a natural setting for the TCSPC technique.

As mentioned in the introduction, a further requirement of DOT is that measurements need to be made at a plurality of positions around a medium on its boundary to obtain an interior image thereof. Several configurations [46], some depicted in Figure 5, are possible for achieving such multiview detection. This, however, poses significant challenges both to the optomechanical design of a DOT scanner and to TCSPC because one needs several detection channels working in parallel to carry out the measurements in reasonable time. Recent advances in parallel multichannel TCSPC make it possible nowadays to carry out such multiview detection at high temporal resolution and exquisite sensitivity. This will be discussed further later, and it will be seen that multichannel TCSPC is a critical technology for the success of TD-DOT.

An important feature of TCSPC is that it allows measuring full TPSFs, which we believe is crucial in exploiting the full potential of TD measurements in image reconstruction algorithms to improve the spatial resolution and quantification achieved by DOT [38]. Moreover, measuring full FTPSFs is a necessity for extracting lifetime.

\section{Instrumentation for Small Animal DOT and the First-Generation TomOptUS Scanner}

The TomOptUS group has developed a first-generation prototype of a TD-DOT scanner for small animal imaging. The purpose here is not to go through all the details of that scanner as this has already been done elsewhere [26], but rather to provide an overview of its architecture and hardware and review some recent results obtained therewith. 
This will lead us in the next section to the needs for the realization of second-generation scanners and the work we have undertaken towards that end.

To put this into a better perspective, we first now briefly review other approaches and systems that have been developed for small animal time-resolved (i.e., time-domain and frequency-domain) DOT imaging (the discussion on systems will be restricted to those for tomographic 3D imaging and excludes systems solely for bioluminescence tomography, as bioluminescence provides by nature a $\mathrm{CW}$ signal). The advantages of TD measurements over CW measurements have been previously discussed as regards information richness. By performing frequency-domain (FD) measurements, it is in principle mathematically possible to obtain the same information as with TD measurements since both are related by a Fourier transform. FD systems resort to a modulated light source to measure the modulation index and phase shift between the light injected into a tissue and the light emerging therefrom after its propagation therethrough. Such systems are limited in small animal imaging owing to the small tissue volumes involved requiring source modulation at frequencies above $1 \mathrm{GHz}$ to obtain sufficient phase shift contrasts [47]. Modulating light sources at such high frequencies is technically difficult and is limited to around $2 \mathrm{GHz}$ (this is what is usually found in the litterature). It is thus in practice impossible to measure the full Fourier spectrum and hence get FD data that is equivalent to TD data; some information is lost. This is a reason why TD measurements better allow reaching the full information that can be obtained from measurements on a tissue with light. This is in fact an aspect that motivates our work.

Notwithstanding the previous discussion on TD versus FD and CW measurements, owing to lower costs, most small animal imaging systems resort to CW measurements. Noteworthy is that commercial systems with tomographic capabilities exclusively resort to CW measurements [4851]. An in-depth broad review of commercial scanners has been provided by Leblond et al. [23]. Regarding research prototypes, most acquire CW data [52-60] and a few are FD systems [56, 61-64]. Interestingly, a study comparing $\mathrm{CW}$ and FD systems and image reconstruction suggested that FD measurements may result in superior images but at much higher costs and efforts as regards instrumentation and computational resources [65].

Regarding systems exploiting TD signals, which is the focus here, the most important characteristics are the temporal resolution and the acquisition time. Acquiring TD data requires more time than $\mathrm{CW}$ data and that is a major issue. Another issue is the cost of TD instrumentation as will be discussed later. Temporal resolution can be characterized by the full-width at half maximum (FWHM) of the instrument response function (IRF) which is measured by directly injecting light from the system's pulsed laser source into a detection channel.

Our systems described herein, through the free-space optics design for noncontact measurements, the short pulse laser along with the detectors, and TCSPC electronics used show unprecedented temporal resolution for multiview dualwavelength TD measurements: IRF $\approx 200$ ps FWHM on average for the first-generation scanner (Section 4.5 provides more details on this) and IRF $\approx 55$ ps FWHM on average for the second-generation prototype (Section 5.4). For comparison, an 8-channel system with an IRF of 260 ps using TCSPC, MCP-PMTs, and optical fibers has been reported [66]. In other work, system of 5 dual-wavelength detection channels has been developed to be used in conjunction with a commercial X-ray CT scanner [67]. The detection channels use optical fibers and red-enhanced PMTs, achieving a temporal resolution of $\approx 465$ ps. To our knowledge, the only TD system that has surpassed the temporal resolution of our first-generation system was a single view single detector transillumination noncontact free-space optics system [36] (IRF 163 ps FWHM) resorting to a supercontinuum pulsed fiber laser (pulse width $\approx 30 \mathrm{ps}$ ) and TCSPC with preselected PMT modules for temporal resolution (150 ps). This system, which measures a single wavelength at a time, is an adaptation of an earlier system that used a time-gated intensified CCD (ICCD) camera with 200 ps gate widths and 25 ps temporal step sizes $[68,69]$. Similar transillumination ICCD systems have been developed for fluorescence lifetime tomography with minimum 300 ps gate widths [70] or a reported IRF of 300 ps FWHM [71]. The systems cited thus far resort to sequential pointwise laser illumination scanned over the surface of the animal to inject light at different locations as needed for tomography. Such scanning is generally timeconsuming.

An alternative is to use wide field illumination with different spatially modulated patterns of light sequentially projected onto the surface of the object to be imaged and to measure the transmitted spatial light pattern [72]. This has been adapted $[73,74]$ for TD small animal imaging using a short pulse laser, an array of micromirrors to generate the illumination patterns, and a time-gated ICCD camera for measuring the temporal signals (200-300 ps gate widths and 25-40 ps temporal step sizes).

To further highlight the unique features of our systems compared to others, ours (1) possess the most dualwavelength channels that can acquire signal simultaneously, this with the best temporal resolution leading to less signal distortion and (2) are able to perform both backscatter and transillumination measurements.

4.1. Architecture Overview. The first-generation scanner, depicted in Figure 6(a), provides at-a-distance measurements without contact with the animal. It comprises 7 dual-wavelength detection channels separated by $40^{\circ}$ for both intrinsic and fluorescence multiview parallel detections. Figure 6(c) shows one such channel (optical design was carried out with the Zemax ${ }^{\text {TI }}$ optical design software, whereas mechanical design of the scanner was carried out with the SolidWorks ${ }^{\mathrm{Tu}}$ CAD software). The channels are mounted on a turntable allowing detection at all angles around the subject, except for a small blind range of $\pm 15^{\circ}$ around the laser beam (Figure 7). The configuration allows both epi-illumination and transillumination, along with intermediate cases (refer back to Figure 5). 


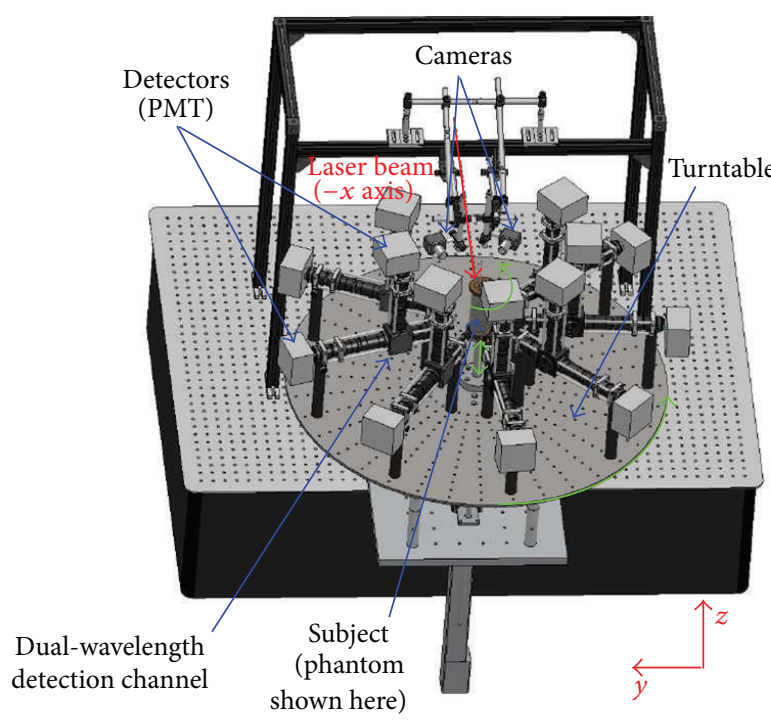

(a)

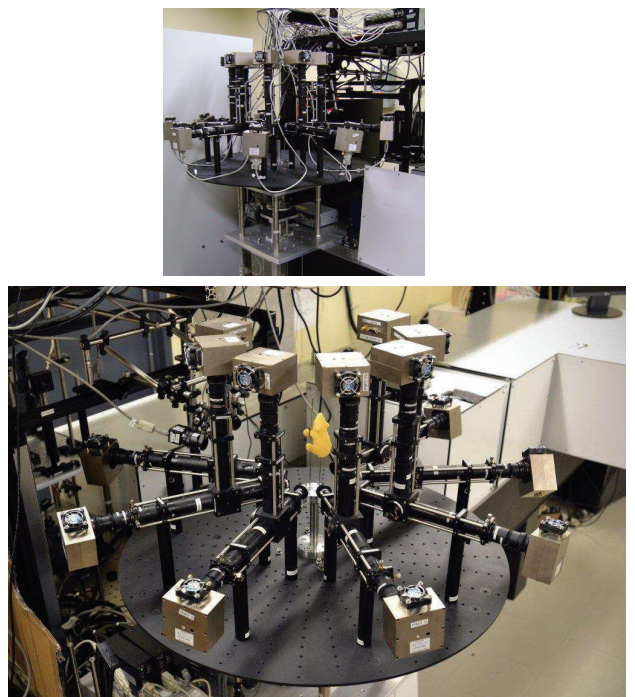

(b)

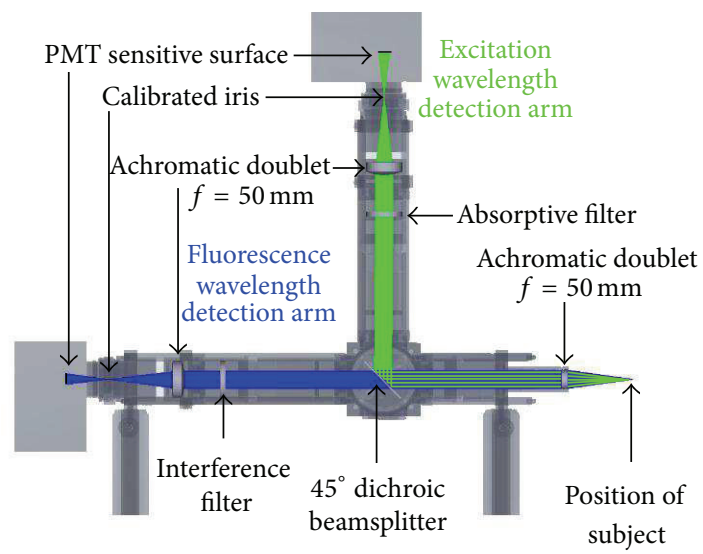

(c)

FIgURE 6: Drawing and photographs of the first-generation TomOptUS scanner (a, b); drawing of one dual-wavelength detection channel (c).
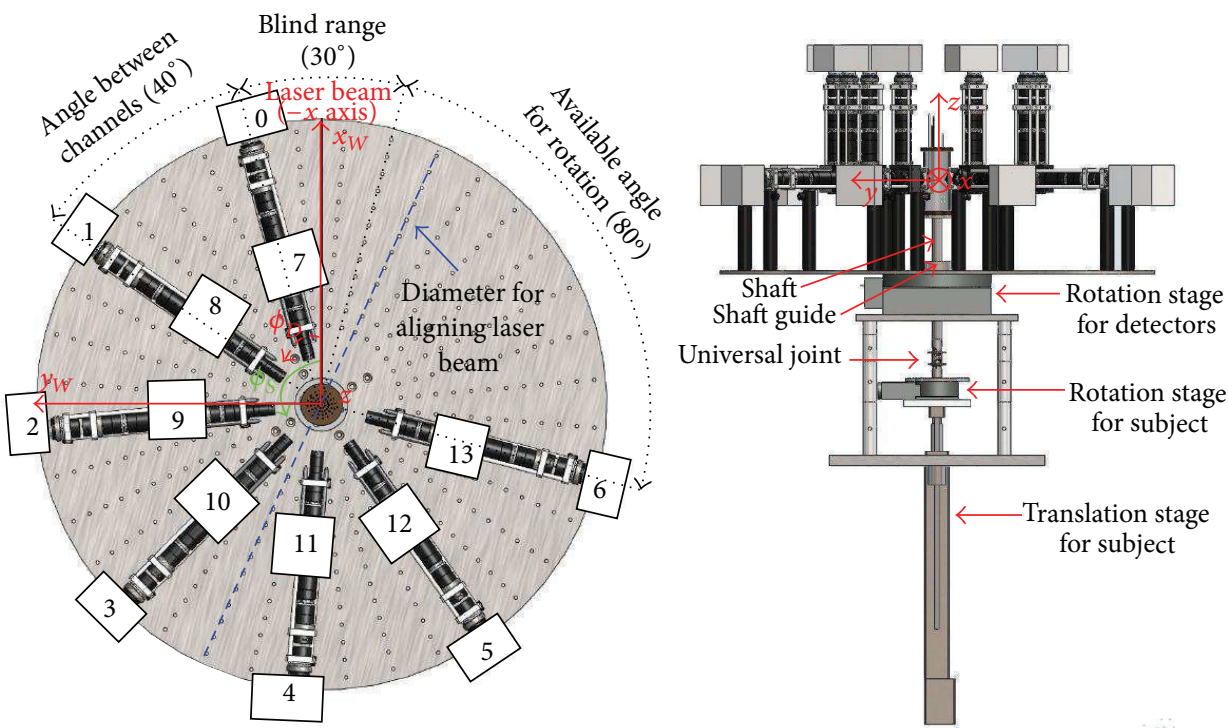

FIgURE 7: First-generation scanner. Drawings of top and side views. 
In this generation, the subject is imaged at the vertical. It can be translated vertically using a motorized translation stage and can also be rotated independently from the detectors around the vertical axis so that the light beam from a laser can be injected anywhere around it. This configuration reduces hardware complexity, which was important to develop a first prototype, compared to a configuration where the subject would be imaged lying horizontally as the latter would require the laser beam to be rotated around the subject. Vertical rotation avoids physiological changes and anatomical displacements within the subject due to gravity.

The scanner is endowed with two cameras for $3 \mathrm{D}$ stereo computer vision to measure the subject's outer surface shape. This is necessary in a noncontact architecture since the surface determines where light propagation changes from diffuse (in biological tissues) to straight line (in air). This allows fixing the boundary conditions to be obeyed by the forward model used to compute the light distribution inside the subject, an essential part in any DOT image reconstruction algorithm. In distinction to other scanners, the TomOptUS scanner uses the same laser beam for both surface and DOT measurements. This allows simultaneous surface and DOT measurements, cutting down on the acquisition time, while reducing system complexity. Additionally, this allows measuring the exact positions where laser light is injected into the subject. These are also required by any forward model used in DOT image reconstruction [75].

4.2. Electrooptics Hardware for TCSPC. To perform TCSPC measurements, the excitation light source used is a modelocked Ti:Sapphire laser (Tsunami, Spectra-Physics, USA) emitting ultrashort light pulses (pulse width 4 ps FWHM) at a repetition rate of $80 \mathrm{MHz}$. This is an ideal light source for a prototype scanner since its emission wavelength can be tuned in the range from 700 to $1000 \mathrm{~nm}$ which is best suited for imaging biological tissues as mentioned earlier and also since it provides ample power at any wavelength in a high quality $\mathrm{TEM}_{00}$ mode circular beam. The laser's tunability also allows high flexibility to image different NIR fluorescent probes. In our system, laser beam steering is carried out as much as possible with reflections of optical components to minimize temporal distortion.

On the detection side, as mentioned previously, high sensitivity and high temporal resolution are needed for measuring the short and faint TPSFs typical of small animal imaging. This calls for TCSPC, which is a technique exactly suited to such conditions [76]. Specifically, we use an SPC134 stack of 4 PCI computer plug-in cards (Becker\&Hickl $\mathrm{GmbH}$ (bh), Germany), with each card achieving an electrical temporal resolution of 6.6 ps FWHM. The 14 detectors used are PMC-100-20 PMT modules (bh) with a spectral response curve in the wavelength range from 300 to $900 \mathrm{~nm}$, having a nominal transit time spread (TTS) of $\approx 180$ ps FWHM (thermoelectric coolers (TECs) are incorporated to reduce dark counts), and with a photocathode diameter of $8 \mathrm{~mm}$.

The reference trigger electrical pulses for the TCSPC cards are derived from the laser pulses with a fast PIN photodiode (PHD-400 N, bh) connected to the STOP input of the TCSPC cards operated in reversed START-STOP mode. Four routers (HRT-41, bh), one for each card, allow connecting up to 4 PMT modules to each card for a maximum of 16 detection channels. An electronics box developed inhouse allows operating up to 16 PMT modules: it supplies and controls power to the PMT modules and their TECs. The box also provides hardware shutdown of the modules to prevent overload light fluxes from damaging the PMTs. Careful optomechanical design of the detection channels prevents optical reflections within them, thus avoiding the TPSFs from being contaminated by such reflections.

4.3. Software. The custom software for operating the scanner, initially developed in LabVIEW ${ }^{\mathrm{m}}$ [26], has completely been redesigned and rewritten in the $\mathrm{C}++$ language for better structure and greater flexibility. Open-source tools have been used throughout: NetBeans and the gcc compiler for Windows for software development and Qt for the graphical user interface (GUI) and its libraries for communications (USB, serial) with the hardware. The GUI allows configuring the scanner's internal hardware settings (TCSPC parameters, number of active detectors, etc.), along with parameters related to an imaging session (ranges and step sizes of subject motion, TCSPC collection time, and laser beam attenuation). The software allows completely automated operation of the scanner during experiments along with complete manual control, which is useful for development and testing purposes and for preliminary data acquisition for setting up an experiment. The GUI also displays the following information in real time as measurements are in progress: actual position of subject and detectors, elapsed time of experiment, photon counting rates, and TCSPC histograms (TPSFs).

4.4. Results Obtained with the First-Generation Scanner. The first-generation scanner has recently allowed developing entirely new techniques for imaging the scattering properties of turbid media mimicking biological tissues [33] and for localizing fluorescent point-like inclusions therein [34]. These techniques critically rely on the high temporal resolution and exquisite timing accuracy and stability of TCSPC which is at the core of our scanner. They will now be briefly reviewed and illustrative results will be presented.

4.4.1. Intrinsic Imaging with Diffuse Photons Density Wavefront Speed. As mentioned earlier, snake photons suffer few scattering events. Because of this, they can to a good approximation be considered to propagate along straight paths (called rays) diverging in all directions from where they originated, that is, where light was injected in the medium (Figure 8 ). The collective propagation of snake photons originating from a given point inside a medium or at the boundary thereof defines an expanding diffusive wavefront (a socalled diffuse photon density wavefront (DPDWF)) therein [33]. When the medium is homogeneous, this wavefront is spherical [34] but gets distorted in a nonhomogeneous medium [77].

The time of arrival of a DPDWF at a given point on the boundary, which corresponds to the arrival time of early 


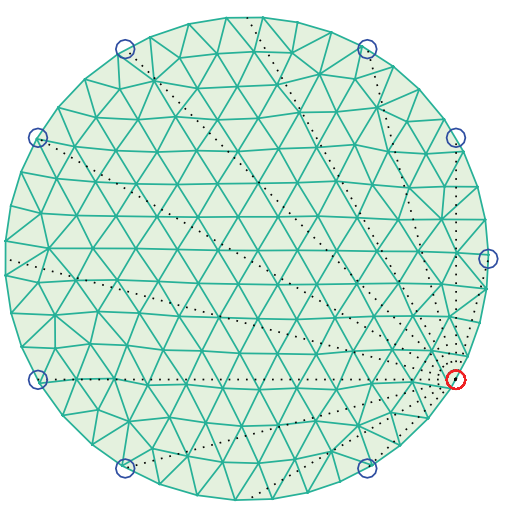

FIGURE 8: Planar section of a cylindrical medium partitioned with triangular elements. Light is injected at the red point and detection positions are shown in blue. For each measurement, a ray (dotted black) is traced between the corresponding detection position and the injection point.

photons (so-called early photons arrival time (EPAT)), can be obtained from a measurement of the TPSF thereat. In fact, as snake photons arrive early, they contribute to the rising edge of a TPSF, and the time of arrival of that edge is taken as the time of arrival of early photons (i.e., the EPAT), which can be stably and reliably obtained from a measured TPSF using numerical constant fraction discrimination (NCFD) previously introduced by our group [78].

The EPAT obviously relates to the speed of propagation of the wavefront inside the medium. As we have shown with our scanner, the EPAT depends on the optical properties of a medium [26], and thus the speed of DPDWFs also depends on those properties since the speed is the distance traveled over the time of travel, with the latter being the EPAT.

These considerations lead to the possibility of reconstructing DPDWF speed maps in order to image the intrinsic properties of media. Such maps have indeed been obtained, showing sensitivity to variations in the optical properties of a medium [33]. Thus far, this has been achieved for variations in the scattering coefficient, and we are currently developing a similar approach for obtaining contrast to variations in the absorption coefficient as well.

Briefly, to obtain a speed map (full details can be found in a previous paper [33]), the medium is partitioned into small cells as depicted in Figure 8 (triangles are chosen for simplicity, but it could also be other shapes). Choosing the cells sufficiently small, the speed within each cell can be assumed constant. Consider a ray, such as one shown in Figure 8, defined by a laser injection position and a detection position (a so-called source-detector (SD) pair). The total time of travel of early photons along that ray (i.e., the measured EPAT) is given by the sum of the individual times spent in each cell intersected by the ray, with the time within each cell being simply the length of the ray intersecting the cell divided by the speed in that cell. Mathematically, this can be expressed as a sum extending over all cells of the partition as

$$
t_{i}^{\mathrm{EPAT}}=\sum_{k=1}^{N_{e}} \frac{d_{i k}}{v_{k}}
$$

where $N_{e}$ is the total number of cells in the partition, $d_{i k}$ is the distance crossed by ray $i$ within cell $k$ of the partition, and $v_{k}$ is the DPDWF speed within cell $k\left(d_{i k}\right.$ is set to 0 for cells not crossed by the ray). Measuring EPATs for a large number of different rays (i.e., SD pairs) leads to a system of linear equations such as (2) that can be solved in the unknowns $1 / v_{k}$ which are then mapped back onto the partition to obtain a speed map. Figure 9 displays reconstructions of such maps from experimental data showing speed contrast where inclusions with scattering coefficient differing from that of the otherwise homogeneous background were inserted (see figure caption for details). The big advantage of this imaging approach exploiting EPATs is that since straight line propagation is considered, image reconstruction of a medium can be carried out slice by slice as in X-ray CT, in contrast to other approaches developed in DOT which necessitate considering the medium as a 3D entity. This makes reconstruction with this approach much faster and also relatively easy to implement as only linear equations need to be solved. Perhaps a disadvantage of this approach is that it relies on detecting snake photons and this may involve low signal-to-noise ratios. This is not a problem with our first-generation scanner owing to single photon detection capabilities and the high light collection efficiency of the detection channels thanks to the large photosensitive area of the PMTs used ( $8 \mathrm{~mm}$ diameter). As will be seen later, this may, however, degrade results with smaller diameter single photon detectors. This calls for optical design optimization as will be discussed.

\subsubsection{Fluorescence Localization by Time-of-Flight Measure-} ments. We have developed an algorithm for localizing in 3D a plurality of fluorescent inclusions located therein by using distance ranging. This is based on the measurement of fluorescence EPATs at different positions around a turbid medium (e.g., a biological tissue) following ultrashort laser pulse illumination at a given position, with this being repeated for several laser injection positions. Such an algorithm is relevant, for instance, to locate small tumor masses or metastatic cells targeted by a fluorescence labeling agent [25].

Referring to Figure 10(a), the time to obtain a fluorescence signal at a given detection position $D$ following the injection of a laser pulse at another position $S$ is given by (i) the time $T_{2}$ taken for the laser pulse (which becomes a DPDWF inside the medium) to travel from the injection point to the fluorescent point-like inclusion at $P$ plus (ii) the time $T_{3}$ for the fluorescence wavefront pulse ensuing the excitation of the inclusion by the laser DPDWF to propagate from the inclusion to the detection position $D$. For a given $S D$ pair of laser injection and detection positions, the time $t_{\text {meas }}=$ $T_{2}+T_{3}$ is what can be measured by a time-domain system. Not considered are the other propagation times indicated by 


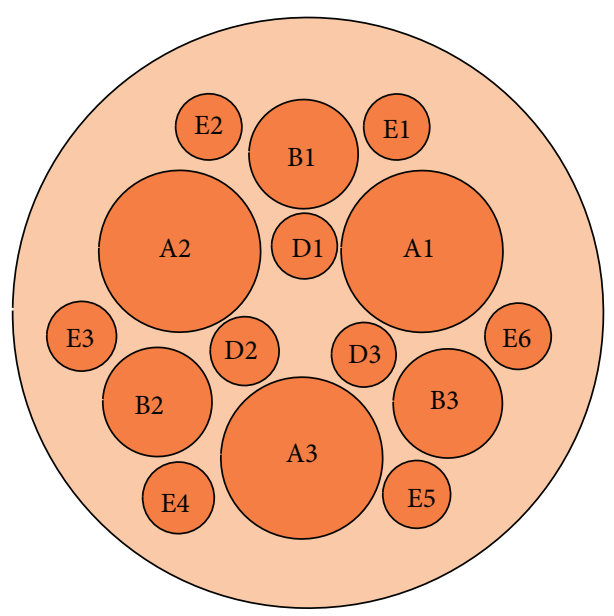

(a)

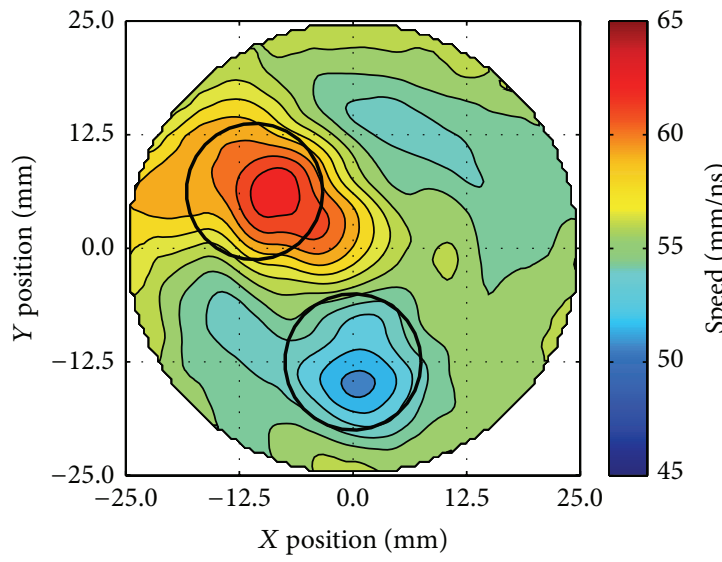

(b)

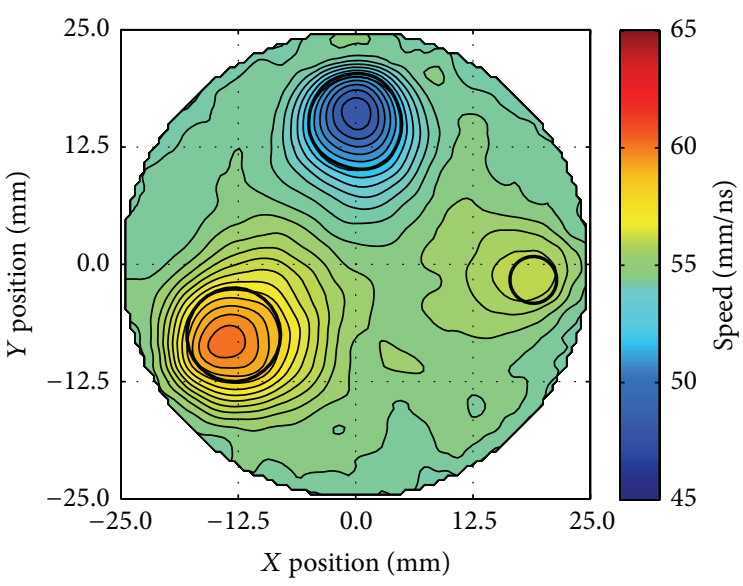

(c)

Figure 9: (a) Top view of phantom used in experiments for obtaining speed maps. Inclusions labeled A, B, D, and E of different sizes and at different locations can be inserted in the medium for obtaining regions with scattering coefficient differing from that of the otherwise homogeneous background. In the results given in (b) and (c), the background's reduced scattering coefficient has value $\mu_{s}^{\prime}=5 \mathrm{~cm}^{-1}$. (b) Speed map obtained with inclusion A2 having $\mu_{s}^{\prime}=2.5 \mathrm{~cm}^{-1}$ and A3 with $\mu_{s}^{\prime}=10 \mathrm{~cm}^{-1}$. (c) Speed map with inclusions B1, B2, and E6 having, respectively, $\mu_{s}^{\prime}=20 \mathrm{~cm}^{-1}, 2.5 \mathrm{~cm}^{-1}$, and $2.5 \mathrm{~cm}^{-1}$.

$T_{0}, T_{1}, T_{4}$, and $T_{5}$ in Figure 10(a) (the time for an electrical signal to propagate in the system's cables, the propagation of light in air and in the optical channels, etc.), since these can be calibrated out as explained in [26,34]. Measuring the time for several SD pairs allows localizing the inclusion as depicted in Figure 10(b). This relies on the fact that different detectors will not measure the same time, with the time for a given detector being dependent on the depth of the inclusion relative to that detector. More precisely, as Figure 10(c) shows, the time measured for a given SD pair defines an oval with

$$
t_{\text {meas }}^{\mathrm{EPAT}}=t_{\mathrm{LP}}+t_{\mathrm{PD}}=\frac{d_{\mathrm{LP}}}{v_{\mathrm{ex}}}+\frac{d_{\mathrm{PD}}}{v_{\text {fluo }}},
$$

where $v_{\text {ex }}$ and $v_{\text {fluo }}$ are, respectively, the speeds of DPDWFs at the laser excitation and fluorescent wavelengths (should the speeds $v_{\text {ex }}$ and $v_{\text {fluo }}$ be equal, then the oval would be an ellipse as the sum of the distances $d_{\mathrm{LP}}$ and $d_{\mathrm{PD}}$ would then be a constant). This oval defines the locus on which the inclusion is to be found, since all points on such an oval will give the same measured time and this is the sole information available from a measurement associated with an SD pair. The arguments thus far have been expounded in 2D for simplicity, but they are equally valid in 3D whereby ovals become ovoids. In $2 \mathrm{D}$, two ovals are in principle sufficient to find the position of an inclusion, with that position being the intersection of the ovals. In $3 \mathrm{D}$, one needs to find the intersection of 3 ovoids. In the case where multiple inclusions are present in the medium, then more ovals (ovoids) must be obtained, which amounts to have more SD pairs of EPAT measurements. In practice, we do not find intersections of ovals (ovoids) but rather trace all ovals associated with SD pair measurements and the regions of highest density in the maps thus obtained correspond to inclusions positions. This is shown in Figures 11(a) and 11(b) in the case of a simulation, where (a) displays a density map and (b) displays that same 


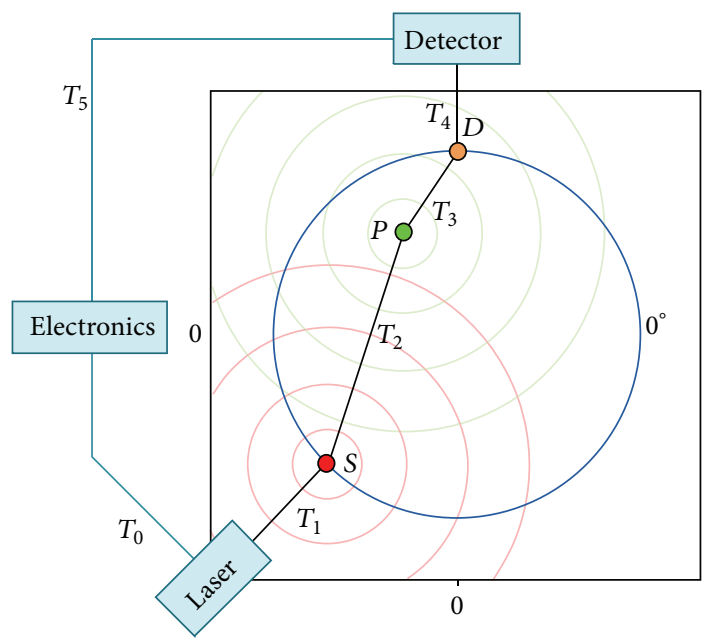

(a)

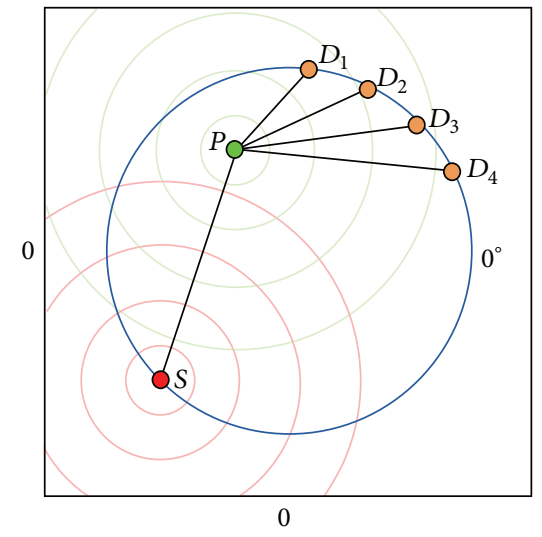

(b)

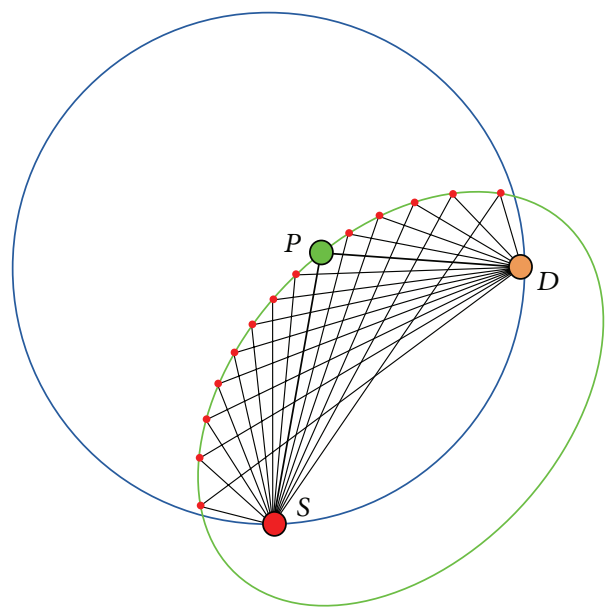

(c)

FIgURE 10: (a) Times and delays involved in an EPAT measurement for a given source-detector (SD) pair. (b) Several SD pair measurements for localizing an inclusion. (c) The result of a given SD pair measurement constrains the inclusion to lie on an oval.

map as a height function from which maxima are extracted. Figure 11(c) shows a localization result for a 3-inclusion case (all inclusions in a plane, experimental data), and Figure 11(d) shows the localization of 4 inclusions at various heights in a 3D cylindrical medium, with a worst case error of $1.7 \mathrm{~mm}$ on the localization of one of the inclusions, which for diffuse optical imaging is excellent.

Further details and results on the localization technique summarized here are given in [34]. Considering that typical speeds for diffusive wavefront pulses are on the order of $1 / 10$ th the speed of light in vacuum and that this technique allows localizing inclusions with $\mathrm{mm}$ accuracy, it is seen that, for such a localization approach to succeed, the system providing the necessary measurements must have a very high temporal resolution and an extremely stable timing accuracy allowing extracting reliably the arrival time of such pulses. Our system has enabled such developments. Further features of the localization approach reviewed here are that (1) it is extremely fast since the operations to obtain an image are very simple and (2) it can localize a fair number of inclusions (we have achieved up to 5). Some shortcomings are that it is limited to finding the positions of point-like inclusions; it cannot image smooth distributions of fluorescence and it cannot localize a large number of inclusions.

4.5. Specifications. As mentioned earlier, the temporal resolution is a critical performance index for a TD measurement system. In the case of our scanner with the dual-wavelength channels at the two wavelengths at which it operates, we measured IRFs FWHM at $780 \mathrm{~nm}$ of 195 ps on average with variations from 116 to $285 \mathrm{ps}$, and at $830 \mathrm{~nm}$ this was $169 \mathrm{ps}$ on average with variations from 114 to $256 \mathrm{ps}$.

To give an idea of typical acquisition times with our scanner, it takes $\approx 4.5$ minutes per slice (corresponding to a given value of the height $z$ of the subject) to acquire a set of measurements comprising a total of 144 measurements (36 laser injection points with an angular separation of $10^{\circ}$, 


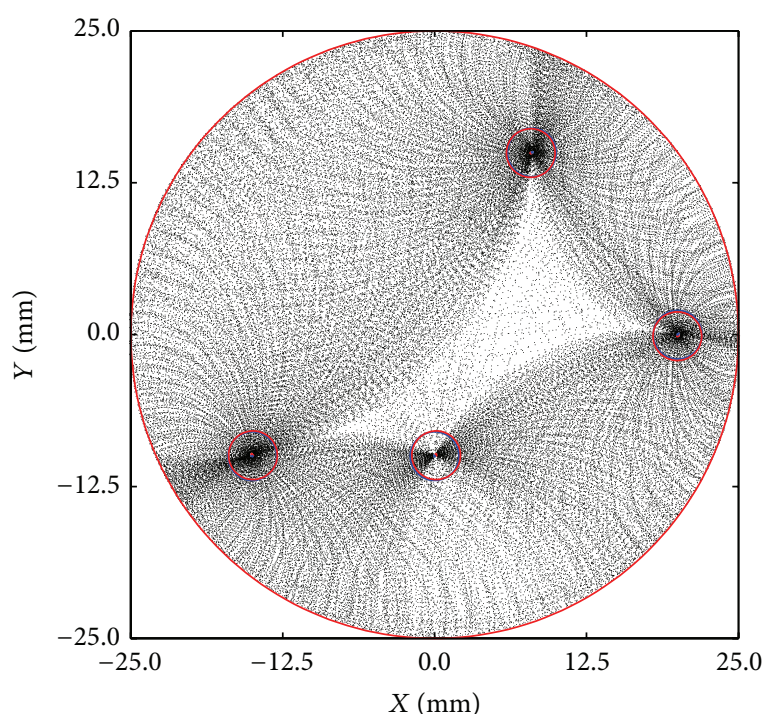

(a)

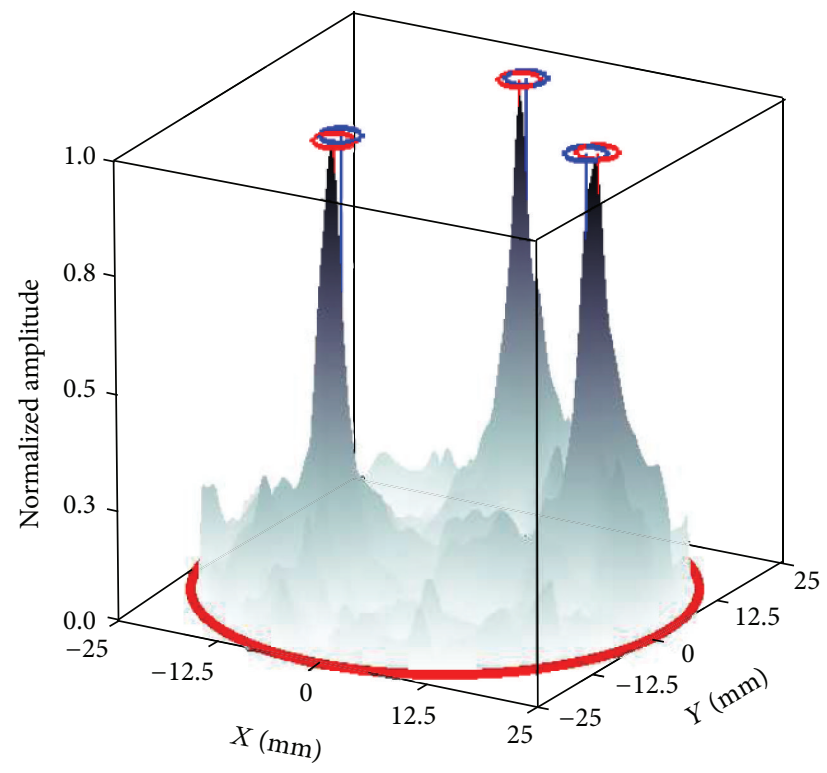

(c)

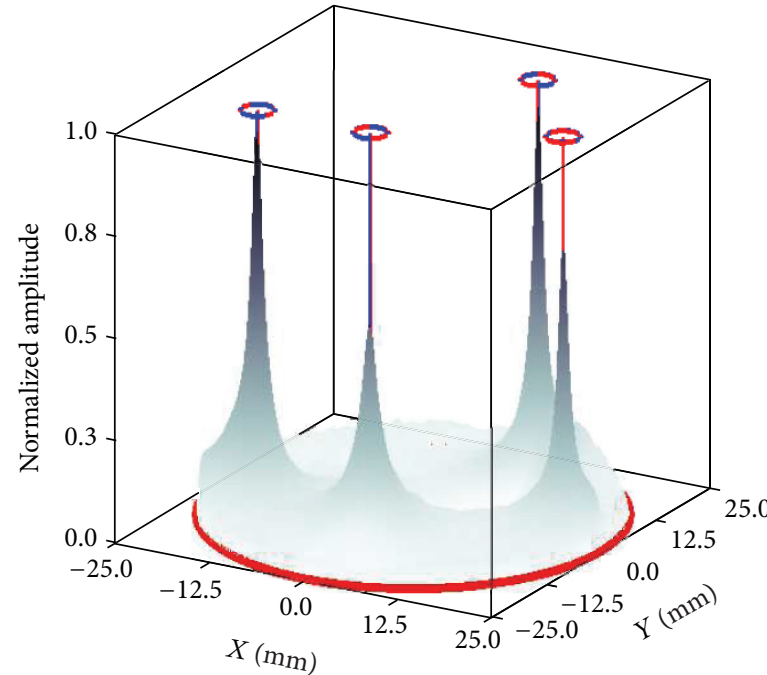

(b)

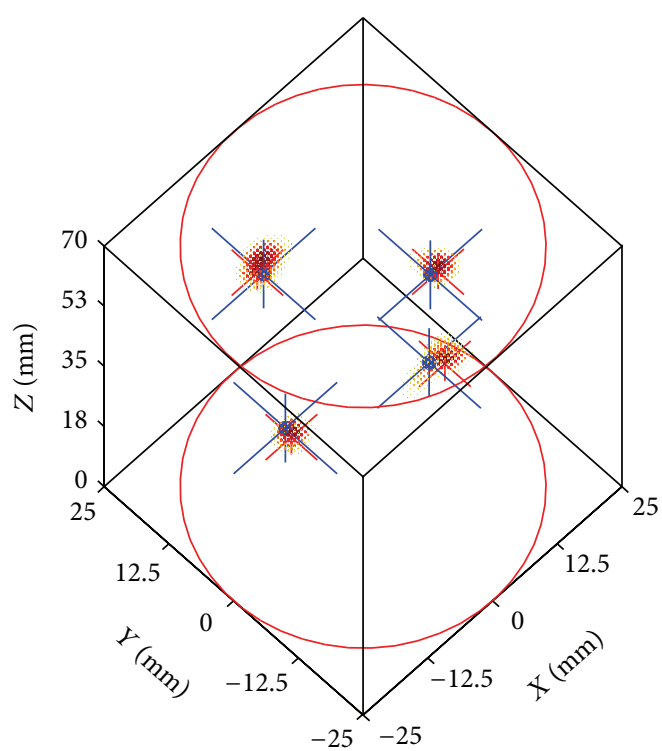

(d)

FIGURE 11: (a) Ovals density map for inclusions all located in a plane (planar 2D case). For all results presented here, red circles show the localized inclusions, whereas blue circles indicate their actual (true) positions. (b) Height function corresponding to (a). (c) Localization result with experimental data for 3 inclusions in a plane. (d) Localization result with experimental data for 4 inclusions in 3D.

detector stepping of $10^{\circ}$ from 0 to $30^{\circ}$, and photon counting collection time of $2 \mathrm{~s}$ ). Such typical acquisition times are very long as this is for only one slice. The bottleneck is the necessity to rotate the detectors. Furthermore, the current scanner is somewhat bulky and resorts to high cost TCSPC electronics, which makes scaling up the number of detectors difficult.

\section{Towards a Second-Generation DOT Scanner}

To deliver an image, DOT reconstruction algorithms require several measurements around a subject, and this needs to be repeated at different longitudinal positions along the subject.
To acquire data in reasonable time for a live subject, measurements will need to be performed faster than what is currently possible with the first-generation scanner. Achieving this requires addressing two major aspects. First, it is necessary to increase the density of detection channels around the subject (i.e., more channels) to avoid rotating the detectors. This necessitates miniaturizing the detection channels by resorting to smaller optics and to replace the bulky PMT modules currently in use with smaller footprint detectors. Second, dealing with more channels requires replacing the expensive TCSPC electronics cards with few channels by more compact denser fully parallel TCSPC electronics at a reasonable cost, which is not currently commercially available. 
Two enabling technologies, to which the SPADlab at PoliMi and its spin-off Micro Photon Devices S.R.L. (MPD) have been leaders in recent years, allow addressing these two major aspects. These technologies, discussed below, are (i) the development of single photon avalanche diodes (SPADs) based on planar silicon technology thin layer junctions [79] and associated front-end electronics circuits $[80,81]$ and (ii) the development of highly integrated electronics for implementing compact parallel TCSPC hardware $[82,83]$.

An alternative to higher detection density and parallel TCSPC could be to use wide field spatially modulated illumination as described in Section 4. Parallelism then comes from the camera and its array of detectors. Such approaches allow injecting more light into a tissue since it is spread over its surface, rather than being concentrated on a small spot (which then requires some upper power limit not to be exceeded so as not to damage the tissue). Wide field illumination thus appears at first sight to offer the possiblity of performing measurements at higher signal-to-noise ratio (SNR) than with TCSPC, hence possibly allowing increasing the acquisition speed. However, CCD cameras are less sensitive than TCSPC which has single photon sensitivity. Also, with time-gated ICCD cameras, the gate must be scanned to different delays for resolving a temporal signal, which requires time. TCSPC in turn acquires full temporal signals by counting and binning the counts in a time histogram (no photons are lost, which makes it more efficient). It is thus not clear which approach in the end will be faster and can achieve higher SNRs. An important question in wide field illumination imaging is to determine the best patterns of light to use for a given imaging task and to reduce their number as much as possible. This can be related to compressive sensing and work on this is underway [84]. Finally, with current technology, the smallest gates are 200 ps wide, whereas SPADs can reach temporal resolutions down to $50 \mathrm{ps}$ and even lower. It is thus seen that two competing technologies can be pursued for TD-DOT imaging, each having its advantages and disadvantages. We favor TCSPC with its higher temporal resolution which we believe will be an advantage in small animal imaging applications.

\subsection{Advances in TCSPC Technologies}

5.1.1. SPADs and Associated Front-End Electronics Circuits. TCSPC requires detectors that can deliver a measurable fast signal in response to the detection of a single photon. Photomultiplier tubes (PMTs) with their high gain have long been the workhorse in this area, but single photon avalanche diodes (SPADs) have become a serious alternative owing to their ruggedness, compact size, higher photodetection efficiency (PDE), and robustness to overloading light signals (whereas a PMT is severely damaged in such cases, a SPAD only saturates).

A SPAD is a pn junction reverse-biased at a voltage $V_{A}$ exceeding the breakdown voltage $V_{\mathrm{BD}}$. It operates in Geiger-mode: due to the strong electric field within the junction, a single charge carrier injected into the depletion layer can trigger a self-sustaining avalanche multiplication process causing the current to rise abruptly (nanoseconds or subnanosecond rise time) to a milliamp range level. When the primary carrier (in fact an electron-hole pair) is generated by a photon, the front edge of the avalanche current pulse tags the arrival time of the detected photon. Since the current continues flowing into the junction until the bias voltage $V_{A}$ is lowered below $V_{\mathrm{BD}}$, a suitable circuit, the so-called quenching circuit, is required. The latter also restores the bias voltage and the device is ready to trigger again.

SPADs. The milestones that led to the development of modern SPAD detectors as pertinent to the present work will now be briefly presented (further details and references can be found in [80]). The first planar silicon technology SPAD structure suitable for monolithic integration was based on an early semiconductor diode structure devised at the beginning of the 1960s [85-87]. Further developments [8892] aimed at improving device performance (reducing the duration and intensity of the diffusion tail which complicated analysis of fast fluorescence decay measurements, reducing the dependence on wavelength of the shape of the temporal response function of the detector, improving uniformity of the PDE over the active area, reducing the dark counting rate (DCR), and afterpulsing probability and their temperature dependence) led to the actual SPAD structure, consisting of a planar double epitaxial structure implemented in a custom technology [79]. Devices are now fabricated starting from an n-type substrate on top of which a p-type epitaxial layer, constituting the SPAD anode, is grown. Finally, an n-type, thin diffusion that constitutes the cathode of the device is implanted. The p-type region has two purposes: to reduce the breakdown value in the central region of the device, avoiding premature edge breakdown and its doping profile is designed to suitably shape the electric field in the multiplication region, with the aim of optimizing device timing response [93]. Eventually, an isolation region can be incorporated, which in conjunction with the n-type substrate forms a well surrounding the device anode. Therefore, by reversebiasing the isolation region-anode junction, it is possible to electrically isolate the SPAD from adjacent devices and to develop monolithic SPAD arrays [94].

The double epitaxial technology, thanks to its compatibility with standard CMOS fabrication techniques, paved the way to further developments allowing directly integrating circuitry onto the detector chip. In the last years, several device prototypes, implemented in standard CMOS technologies, have been reported. However, these devices present a lower PDE $[95,96]$ or a much worse timing resolution [97], a significantly higher DCR per unit area $[98,99]$, and a higher afterpulsing probability with respect to custom SPADs, forcing device designers to use smaller active areas and low excess bias voltages to limit detector noise. On the contrary, optimization of the electric field profile allowed by custom technologies leads to better performance in terms of timing resolution, DCR, afterpulsing (allowing the development of large area SPADs with diameters from several tens of microns up to few hundreds of microns [100]), and higher PDE (further improvements have been recently demonstrated by using a thicker absorption layer [101]). 
Quenching and Resetting. Another important aspect when using SPADs is quenching the avalanche buildup after the detection of a photon $[102,103]$. The main issues here are the speed at which quenching occurs, the hold-off time, and the reset transition.

Quenching should enter in action as fast as possible after an avalanche is triggered to reduce the avalanche charge. The latter is responsible for energy dissipation within the detector, which should be kept low to prevent excessive power dissipation and damage. It also leads to secondary light emission, giving rise to unwanted optical cross talk between detectors. Finally, as the avalanche current proceeds through the detector, some charge carriers are trapped and get released after a random time. Such carriers can trigger a secondary unwanted avalanche (afterpulse).

To speed up quenching, both the delay $T_{d}$ with which the quenching transition starts after the avalanche and the transition time $T_{q}$ must be minimized. With passive quenching circuits (PQCs), the onset of the transition is immediate after the avalanche, but the transition time can be fairly long. Active quenching circuits (AQCs) can speed up this transition, but their feedback loop may introduce a significant delay.

The hold-off time, which is the time the SPAD spends in the quenched state, must be kept short to achieve high counting rates but should not be too short to leave enough time for trapped carriers to be released. Controlling the holdoff time is a feature that can be obtained with AQCs, but not with PQCs.

Following quenching, the SPAD needs to be reset. This requires the bias voltage to be brought back to its quiescent value above the breakdown voltage with a duration of at most a few nanoseconds. Indeed, during the reset phase, a degradation of the photon timing resolution and of the PDE, due to the variation of the SPAD overvoltage, can occur. This calls for minimizing the reset transition time so that the probability of detecting photons during this transition is kept to a minimum. PQCs inherently show exponential recovery, with typical reset time constants of a few hundred nanoseconds. AQCs on the other hand allow faster reset transitions. Owing to the respective advantages of PQCs (faster response to an avalanche) and AQCs (faster transitions and adjustable holdoff time), mixed active-passive quenching is nowadays the preferred approach [103].

Current Pick-Up. SPADs with timing resolutions in the few tens of picoseconds initially had very small photosensitive areas (typ. 10-20 $\mu \mathrm{m}$ diameter). The difficulty in developing optical systems with such small area detectors in terms of optical alignment and light collection led to efforts to develop larger area SPADs [79]. The timing resolution of planar SPADs, however, strongly depends on the diameter: in the very early part of the avalanche, carrier multiplication is confined within a small area and the current rises with small statistical fluctuations. The later part of the current rise corresponds to the progressive spatial spreading of the multiplication process and is subject to higher statistical fluctuations; hence, the larger the active area, the worse the timing performance. The trade-off between active area and timing resolution may be overcome by detecting the avalanche current during the initial part of its rise [104]. With an appropriate current pick-up circuit, a timing resolution down to 35 ps FWHM was demonstrated at room temperature with thin depletion layer SPADs having an active area diameter up to $100 \mu \mathrm{m}$ [105]. Further work on current pickup circuits for their integration near to the detector has made pixel architectures for SPAD arrays and multichannel TCSPC possible $[81,106]$.

5.1.2. Multichannel Parallel TCSPC Electronics. TCSPC relies on periodic excitation of a process (an example is fluorescence emission) with ultrashort pulses of light and measuring the time interval between the arrival of each single detected photon in response to the process (in our example this would be a fluoresced photon) and the excitation light pulse that gave rise to that photon in the process. Hence, each detected photon is time-tagged according to its measured time interval. At high pulse repetition rates in the range of tens of $\mathrm{MHz}$ such as what can be obtained with some solid-state and diode lasers, because of the typical dead time in the processing electronics and possibly in the detectors such as for SPADs, it is impossible to work at photon counting rates where several photons would be detected for each cycle of the periodic excitation. Even, the probability of detecting a photon per excitation cycle must be kept much smaller than 1 (a probability of $\approx 1 \%$ is typically used in practice). Such a low probability is necessary to make the detection of 2 or more photons in the same excitation cycle negligible since all photons arriving after the first one cannot be processed as the electronics are busy with the first. The electronics are thus blind to photons arriving after the first within an excitation cycle, and so if multiple photons occur too frequently, the waveform to be measured gets distorted towards short times. Given that the condition of low probability is satisfied, accumulating a large number of time-tagged photons allows measuring a temporal waveform (such as a fluorescence decay curve) by forming a histogram of the number of detected photons in terms of their time tags. In effect, TCSPC is a means to statistically reconstruct a waveform that is then thought of as a probability distribution (the probability of occurrence of photons as a function of time for a given process).

Hence, apart from detectors and associated front-end electronics for detecting single photons, TCSPC requires processing electronics blocks to build the histogram. In one implementation, this is done with an analog time-toamplitude converter (TAC) that converts the time interval between a START electrical pulse (corresponding to a laser pulse) and a STOP electrical pulse (obtained from the detection of a photon) into an analog voltage proportional to that interval $[41,107,108]$. This voltage is then converted to a digital number with a fast analog-to-digital converter (ADC), and this number is used to increment a counter in a bank of counters at the address corresponding to that number. Each counter thus implements a time bin whose address is proportional to time. After time-tagging and counting a large number of photons, the bank holds the 


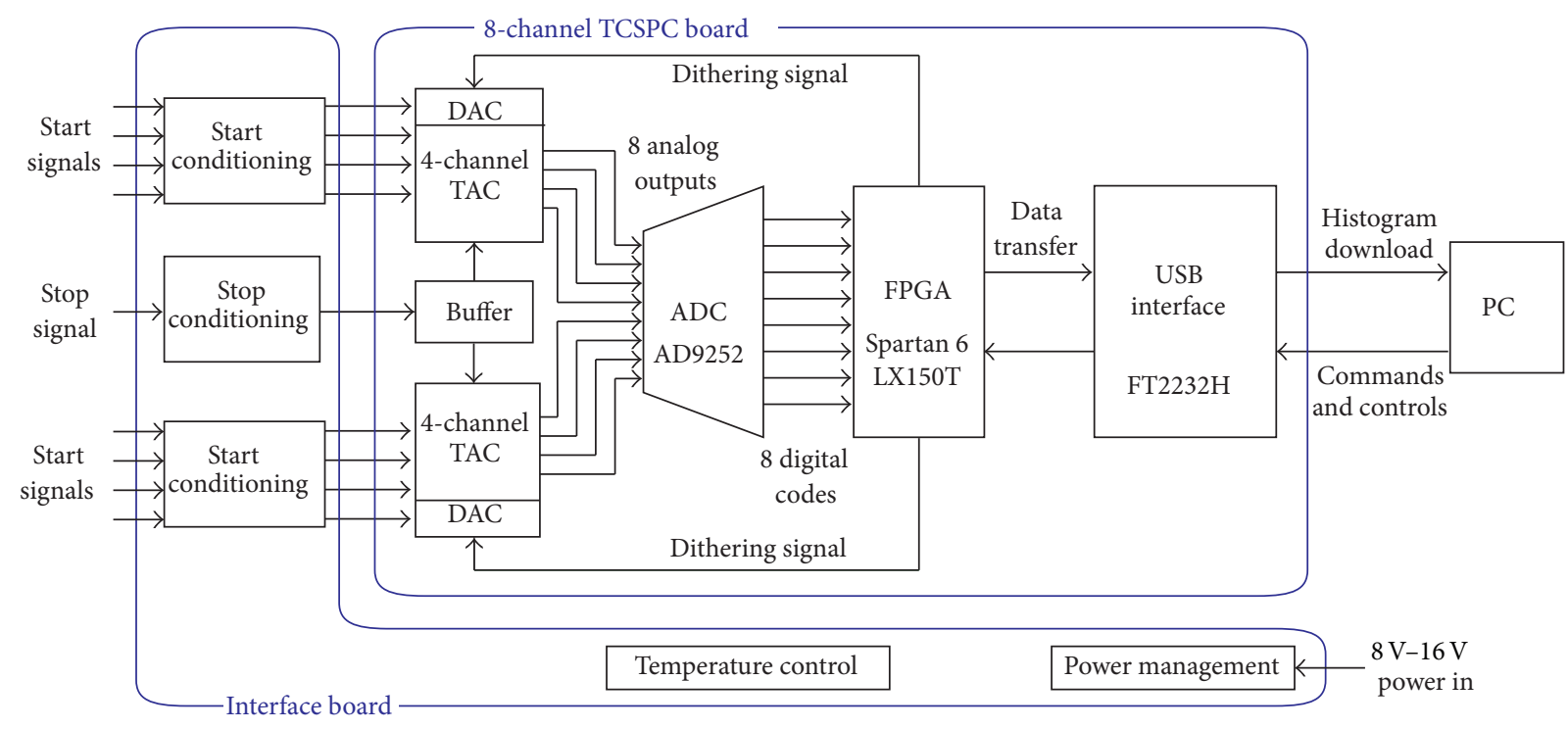

Figure 12: Block diagram of the 8-channel PoliMi TCSPC module (DAC: digital-to-analog converter, TAC: time-to-amplitude converter, ADC: analog-to-digital converter, FPGA: field-programmable gate array, USB: universal serial bus, and PC: personal computer).

histogram corresponding to the signal to be measured. In another implementation of TCSPC, the time interval between a START pulse and a STOP pulse is directly converted to digital form with a device called a time-to-digital converter (TDC). There are several ways to implement a TDC, which exploit the transit time of the timing signal within a chain of logic gates and are typically based on Vernier delay lines [109] or on ring oscillators [110].

Applications of TCSPC require more and more multichannel detection capabilities to increase measurement throughput, and TD-DOT is one example. A relatively high number of detection channels (typically up to 32) can be handled with modules in the form of cards that can be inserted in mother board slots of computers, as the ones used in our 1st-generation DOT scanner. Such modules exhibit high performances in terms of timing resolution (a few picoseconds), linearity, and dynamic range (hundreds of nanoseconds). However, their limited parallel signal processing capability, large size, high cost, and excessive power consumption prevent further scaling-up of the number of detection channels. Thus, integrated circuit solutions [82] able to reach all the required timing characteristics and ensuring cost and size reduction [111] are mandatory for multidimensional imaging systems.

5.2. Eight-Channel TCSPC Module. To fulfill the requirements of modern multichannel TCSPC applications, an 8channel TCSPC module prototype, which can eventually be easily scaled up, has been developed by the PoliMi group. The module is based on an intrinsically multichannel structure, whose detailed description can be found in [83].

The block diagram of the 8-channel module is depicted in Figure 12. An interface board is used to adapt the singleended external timing signals in NIM standard analog format (eight START pulses and a common STOP pulse) to the differential TAC input signals, provide the external power supply to the TCSPC board (the module operates with a single DC power supply delivering from $8 \mathrm{~V}$ to $16 \mathrm{~V}$ ), and perform temperature control (regulating the rotation speed of two fans). The TCSPC electronics themselves are implemented on a $95 \times 40 \mathrm{~mm}$ eight-layer printed circuit board (PCB) to provide several power planes and avoid electrical cross talk between the analog signal conditioning stages and the digital processing blocks.

The TCSPC board is based on two 4-channel integrated TACs directly driving a commercial 8-channel ADC. An on-board FPGA processes the ADC data outputs and the computed values are used to build the TCSPC histograms into the FPGA internal memory. Finally, data are transferred to a PC through a USB link, used also as an interface for remote control. The two integrated TAC arrays, each including four independent converters, are built in $0.35 \mu \mathrm{m}$ $\mathrm{Si}-\mathrm{Ge}$ technology. The 4-channel TAC is an improved version of a converter previously developed at PoliMi [82] which was itself based on a single-channel prototype [112]. Each channel inside the TAC array features a variable full-scale range (FSR) that can be chosen among four values: $11 \mathrm{~ns}, 22 \mathrm{~ns}, 45 \mathrm{~ns}$, and 88 ns. To maximize system flexibility, the FPGA can digitally set this parameter, even when a measurement is running. To reduce the board dimensions, the TAC outputs were designed to match the ADC input dynamic range, so that no external operational amplifiers are required.

To keep dimensions compact, the AD9252 A/D converter (from Analog Devices) was chosen, as it features eight independent channels, a 50 MSPS conversion rate, and a 14-bit data output. Although it satisfies most of the TCSPC requirements, its $\pm 40 \%$ LSB differential nonlinearity (DNL) is too high. To obtain an appropriate DNL, we decided 


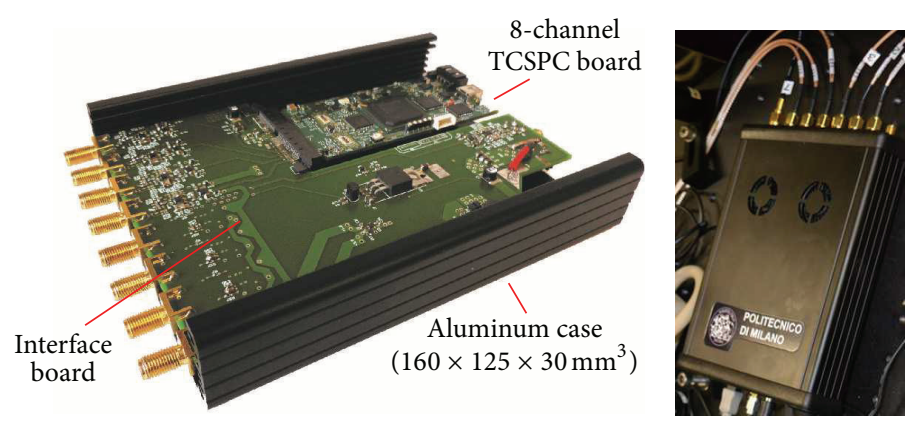

Figure 13: Photographs of the 8-channel PoliMi TCSPC module.

to implement the dithering technique. A random dithering signal is added to the TAC output voltage through a D/A converter and subsequently subtracted from the digital value resulting from the ADC conversion. The dithering technique implemented here is known as sliding scale [113], and we have previously demonstrated its effectiveness in reducing the overall DNL [114]. To minimize dimensions, the adder stages and the integrated D/A converter (based on a 10-bit resolution current-steering segmented architecture $[115,116]$ ) are included in the TAC chip too.

As already described, the ADC outputs are acquired, processed, and stored into the FPGA (Spartan-6 XC6SLX150T from Xilinx) internal memory. At power-on, the FPGA reads the contents of an on-board FLASH memory programming itself automatically, thus making the module ready to use.

The FPGA samples and deserializes the eight ADC serial outputs; each deserialized result is stored in a dedicated internal FIFO memory to decouple the sampled data stream from that for data processing. The FIFO output, after dithering compensation, corresponds to the histogram memory cell whose value can then be updated.

The eight histograms are stored in eight dual-port RAMs divided into $2^{14}$ cells, each having a 32-bit depth. Measurement data are updated by writing into one port and can be simultaneously sent to the $\mathrm{PC}$ by reading the memory from the second port (also used to erase the RAM content). Histogram data, along with additional information useful to characterize the measurement, are exported to an external PC using the FT2232H Hi-Speed 2.0 USB transceiver from FTDI chip.

Photographs of the complete 8-channel TCSPC module are shown in Figure 13. The interface board has been tailored to be enclosed in a small aluminum case $(160 \times 125 \times$ $30 \mathrm{~mm}^{3}$ ). The TCSPC board itself is shaped to be connected into an 80-pin connector. This way the board can be included in the stand-alone 8-channel module as presented or in a system featuring more channels by simply parallelizing more boards [111].

The complete module has a power consumption of about $6 \mathrm{~W}$ and a time bin width ranging from 0.8 to $6.4 \mathrm{ps}$ depending on the selected TAC FSR. Several experiments were conducted on the TCSPC system to evaluate three critical parameters: the time resolution, the DNL, and the cross talk between channels. Besides those, it is important to evaluate the conversion rate of the channels, which depends on the maximum TAC-ADC dead time. A value of $110 \mathrm{~ns}$ was measured for the latter and, considering a maximum ADC conversion delay of $88 \mathrm{~ns}$, a total time of approximately $200 \mathrm{~ns}$ for a single conversion is obtained. Hence, the system reaches a useful maximum conversion rate of $5 \mathrm{MHz}$ per channel.

To thoroughly characterize the module, all channels and all FSRs were independently tested, but, for brevity, only the time resolution and DNL values for one channel for the $45 \mathrm{~ns}$ full-scale range are reported in Figure 14; more details on the performance and on the set-ups used to carry out the measurements can be found in $[83,111]$. The timing resolution that can be obtained depends on the selected FSR. As Figure 14 shows, it is 51 ps FWHM (considering the value at mid-FSR) for the $45 \mathrm{~ns}$ FSR and, in general, it improves as the FSR is reduced: switching from the 88 ns to the 45 ns FSR, an increase by a factor of two can be observed, with a measured FWHM resolution scaling from 110 ps down to 55 ps. As the FSR is further reduced, the resolution improvement factor is lower: the timing resolution for the $22 \mathrm{~ns}$ FSR is $30 \mathrm{ps}$ and, for the shortest range, it decreases to $18 \mathrm{ps}$. On the contrary, the peak-to-peak DNL value is constant and extremely good for all FSRs, being lower than $4 \%$ of the time bin width for almost all time delays. Finally, the cross talk, measured in the worst possible operating conditions [83], resulted in a peak-to-peak disturbance lower than $6 \%$ of the time bin width, extremely weak, and completely negligible in an actual TCSPC acquisition.

5.3. A Second-Generation DOT Scanner Test Prototype. In 2007, the TomOptUS group demonstrated the feasibility, for a single detection channel, of resorting to SPAD detectors to replace more bulky PMTs for DOT measurements [117]. This showed that it was possible to obtain sufficient signal with such detectors despite their much smaller photosensitive area ( $50 \mu \mathrm{m}$ diameter compared to typ. $\approx 8 \mathrm{~mm}$ for PMTs). Since then, other groups have also investigated the possible use of SPADs for diffuse optical imaging measurements [118121]. We have now gone one step further by modifying our 1st-generation scanner described above to integrate SPAD detectors and the compact 8-fully-parallel-channel PoliMi TCSPC module described above. This is to demonstrate to a larger scale the feasibility of miniaturizing the detection 


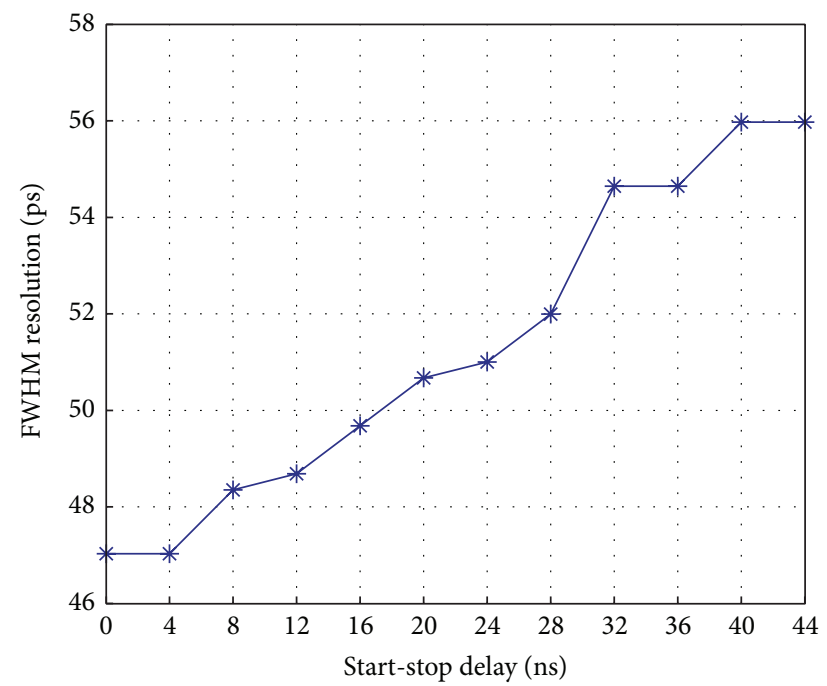

(a)

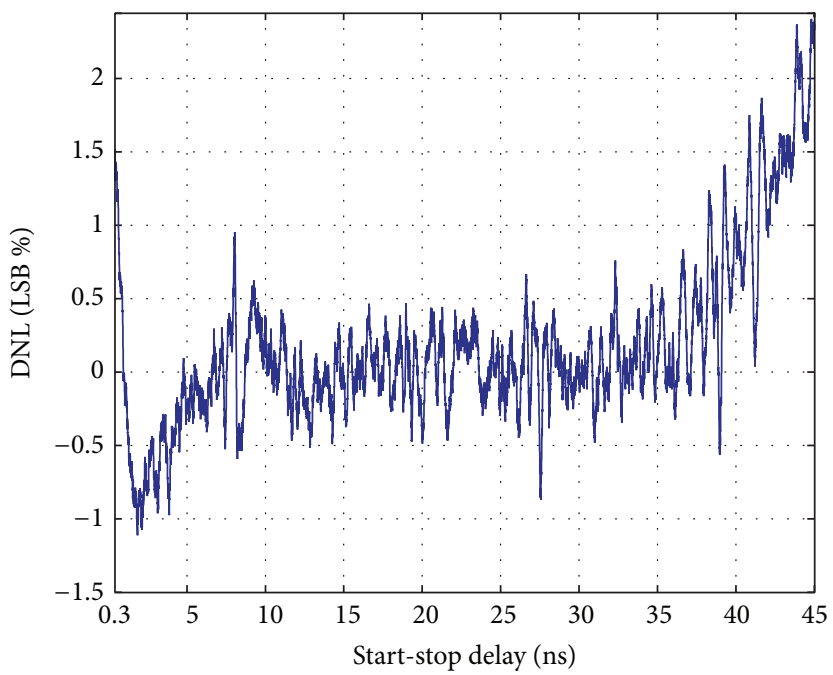

(b)

FIGURE 14: Main performance figures for the $45 \mathrm{~ns}$ FSR. (a) FWHM timing resolution. (b) Differential nonlinearity.

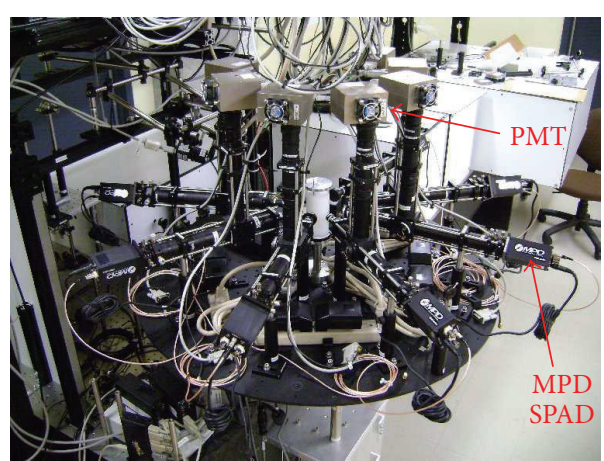

(a)

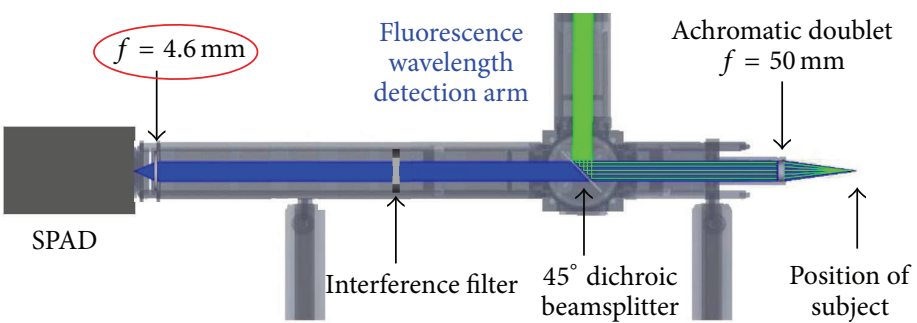

(b)

FIGURE 15: Photograph of the scanner where SPADs replace PMTs in the fluorescence arms of the detection channels (a). Schematic of the fluorescence arm of a detection channel modified for a SPAD detector (b). Essentially, a $50 \mathrm{~mm}$ focal length lens has been replaced by a much smaller focal length lens of $4.6 \mathrm{~mm}$, and the lens tube was made longer.

channels and establish design criteria in view of developing the second-generation scanner with such technologies. This is a first step towards the realization of a high detection density fully parallel TD-DOT scanner, whereby each detection channel will have its own electronics rather than resorting to routers which introduce interchannel cross talk and which also further limit the possible photon counting rate.

More specifically, in the fluorescence channels of the firstgeneration scanner, we replaced the PMTs by SPAD detectors (PDM module, $50 \mu \mathrm{m}$ active diameter, $<50$ dark counts/s with thermoelectric cooling, and 50 ps nominal timing resolution but our modules had better performances with timing resolutions averaging at $42 \mathrm{ps}$ ) from Micro Photon Devices (MPD, Bolzano, Italy) and adapted the optomechanics (lenses and lense tubes) accordingly due to the smaller photosensitive area of SPADs compared to that of PMTs $(50 \mu \mathrm{m}$ versus $8 \mathrm{~mm}$ ) (Figure 15). The PoliMi 8-channel TCSPC module replaces the Becker\&Hickl TCSPC cards for the fluorescence channels. The scanner's software has also been modified to acquire the data from the PoliMi TCSPC module. We chose to establish feasibility with the fluorescence channels, since fluorescence signals are much weaker than intrinsic ones, and thus feasibility with fluorescence detection warrants feasibility for the intrinsic case. Furthermore, for small animal imaging, fluorescence imaging is of greater interest than intrinsic imaging.

5.4. Results: A Comparison with the First-Generation Scanner. We have first characterized the temporal resolution of the fluorescence channels with the SPADs and the 8-channel PoliMi TCSPC module. This temporal resolution directly depends on the timing resolution of the TCSPC electronics and SPADs. We operated the TCSPC module with the FSR set at $22 \mathrm{~ns}$ for which the timing resolution is $30 \mathrm{ps}$ as previously 


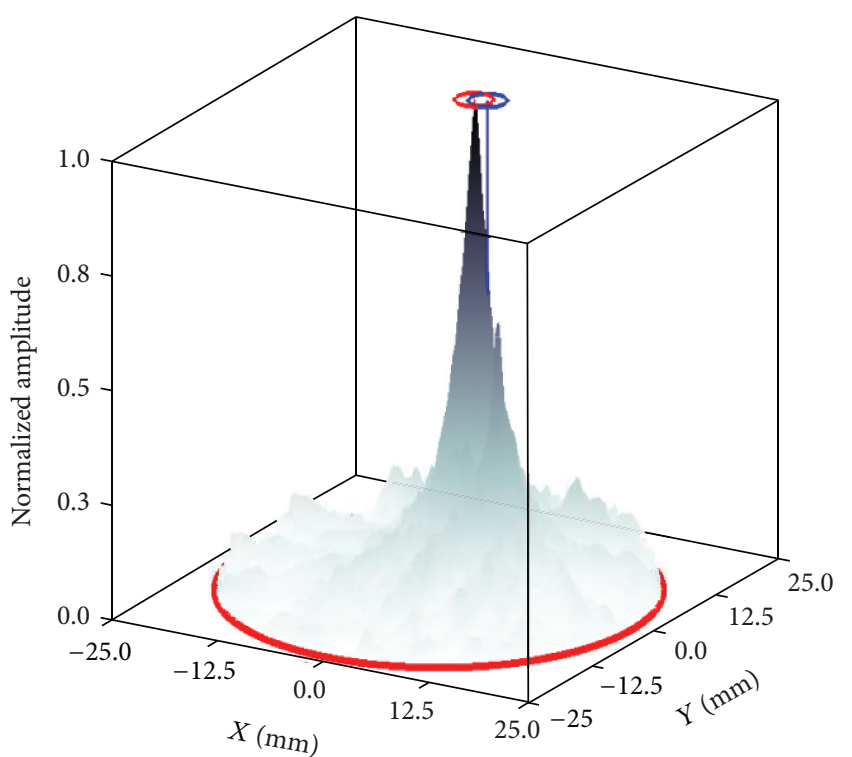

(a)

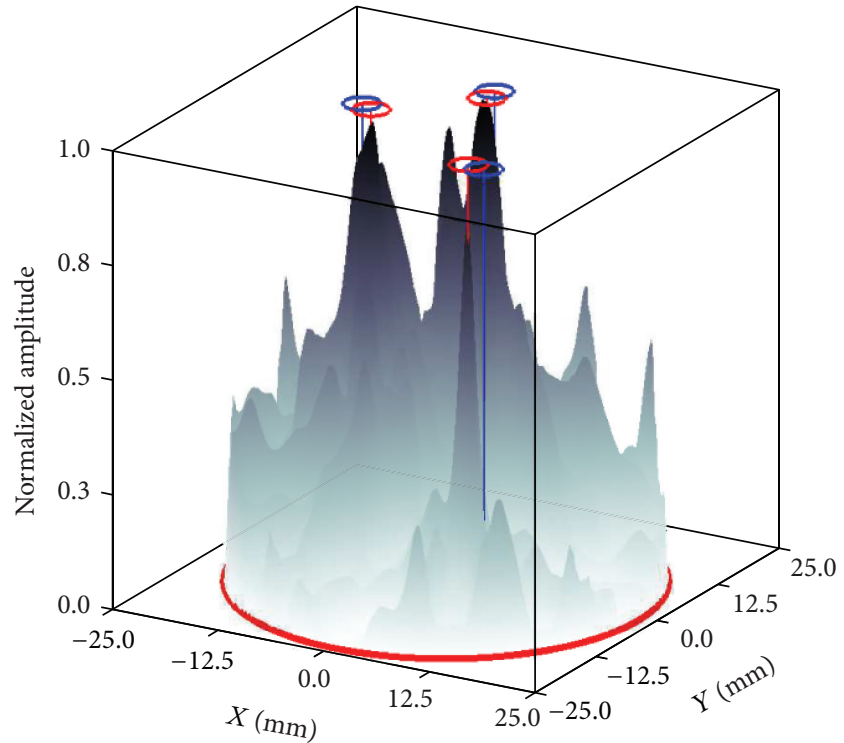

(b)

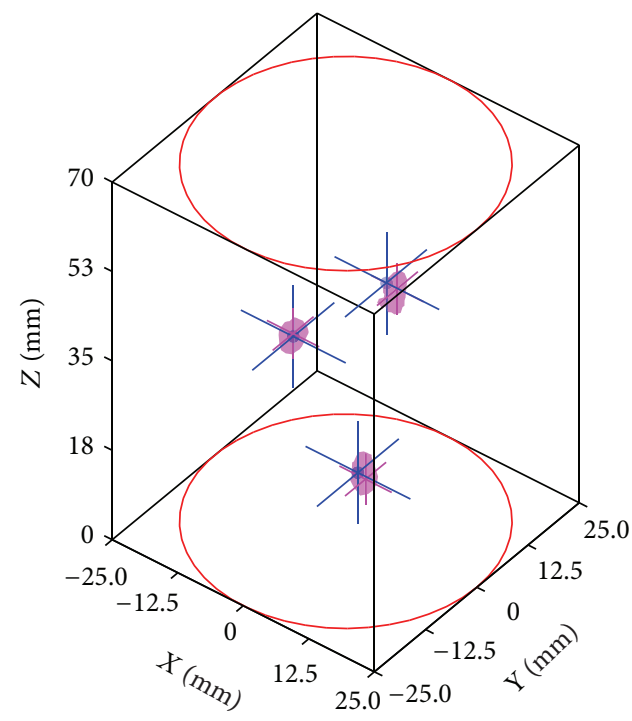

(c)

FIGURE 16: Fluorescence localization results for experimental data obtained with SPADs. (a) One inclusion. (b) Three inclusions in a plane (2D case). (c) Three inclusions in 3D.

mentioned. We obtained an average IRF FWHM at $830 \mathrm{~nm}$ over all 7 channels of 54 ps with variations from 49 to 62 ps. This is a threefold decrease compared to the PMT channels of the first-generation scanner.

We then performed measurements of fluorescence EPATs with the SPADs and the 8-channel PoliMi module to reconstruct fluorescence inclusions localization maps following the approach presented in Section 4.4.2. Figure 16 shows representative results for the localization of 1 and 3 inclusions in a plane (2D case) and 3 inclusions in $3 \mathrm{D}$. It is not the purpose here to go into a detailed analysis of these results but, generally, localization errors are larger when using SPADs compared with those obtained with PMTs (for which
Figure 11 gives a representative example). This can be traced back to the fact that signals with SPADs are weaker with the current optomechanical channel configuration compared to those obtained with PMTs under the same conditions (same photon counting collection time). This calls for an optomechanical rework of the detection channels to obtain a design optimized for SPADs so that light collection can be increased; presently the current versions of the channels for SPADs are mere adaptations of the channels designed for PMTs. Such optomechanical rework is a major challenge as smaller optics will need to be resorted to in order to achieve denser channel packing, while increasing the light gathering power. Furthermore, working with small optical 


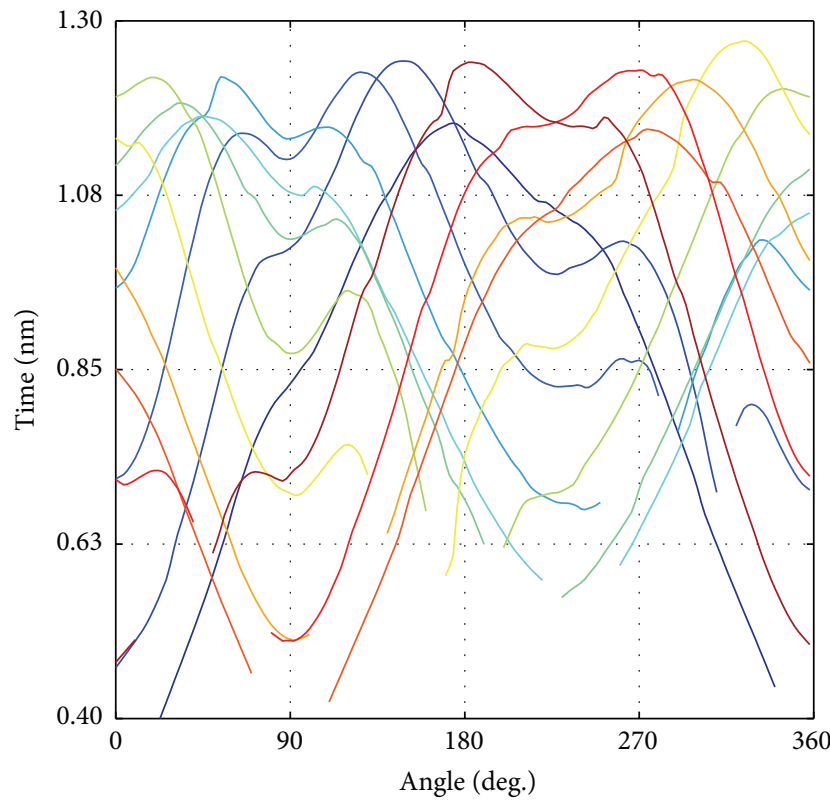

(a)

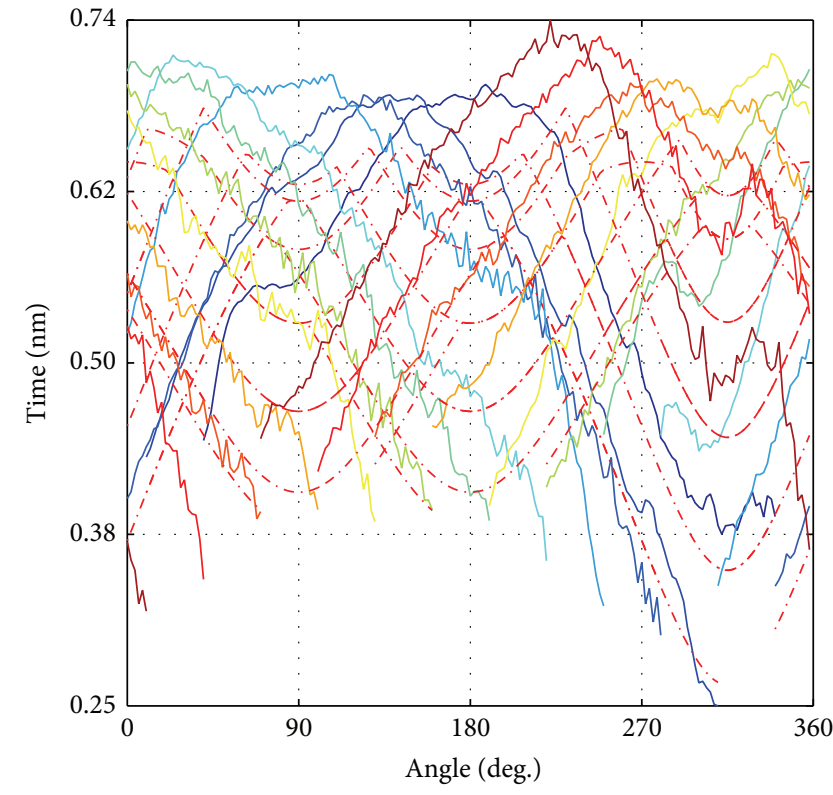

(b)

FIGURE 17: (a) EPAT projections measured with PMTs (these are the projections used to obtain Figure 11(c); each colored curve corresponds to a projection). (b) EPAT projections measured with SPADs corresponding to Figure 16.

elements poses more stingent requirements on alignment and thus on tolerancing. These are major practical issues that will need to be addressed. Ovecoming such difficulties will be a very significant step forward in TD-DOT technology towards reducing data acquisition time, which is a major bottleneck as discussed earlier.

Figure 17 shows typical EPAT projections (also called "sinograms" [34]) acquired with PMTs and SPADs. Such projections serve as data for obtaining localization maps. A given EPAT projection corresponds to the set of EPATs measured for a given point where laser light is injected into the medium; that is, the location of the source $(\mathrm{S})$ is held fixed. As can be seen, EPAT projections obtained with the SPADs are noisier than those obtained with PMTs. This explains why localization errors are larger for the data acquired with SPADs compared to PMTs. Again, this is not a problem with the SPAD detectors but rather with the nonoptimized current optomechanics.

The results presented in Figure 16 clearly indicate that imaging with SPADs to replace PMTs is possible, and we may hypothesize that, with appropriate optomechanical design, the performances of SPAD channels should approach or equal those of PMT channels. The same conclusion is also motivated by past investigations carried out in our laboratory [117]. This is the reason why we are pursuing the development of our scanner with SPADs.

\section{Summary, Conclusions, and Current Work}

In this review, we provided background on the interaction of light with biological tissues to motivate the advantages for resorting to TD measurements in terms of the higher information content in the signals thus acquired. The characteristics of such signals represent challenges related to their measurement. This leads to requirements on the instrumentation necessary to acquire them without disturbing their integrity as much as possible. We then went on to present instrumentation that we developed for small animal DOT imaging (first-generation TD-DOT scanner) based on PMT detectors and TCSPC cards which fulfills these requirements; that is, it is able to acquire the signals and preserve their integrity, but it does not do so in sufficiently short time for practical purposes in small animal imaging. This led us to investigate TCSPC technologies that would allow overcoming this difficulty, which is SPAD detectors and fully parallel multichannel highly integrated electronics for TCSPC. We developed a prototype scanner based on these technologies and demonstrated that they will allow developing next-generation TD-DOT scanners, as we were able to obtain results similar to those with PMTs and TCSPC cards. There is, however, a challenge in this endeavor, which is to optimize detection channels optomechanics for SPADs with their "microscopic" photosensitive areas in order to bring the amplitude of the acquired signals to levels comparable to those obtained with PMTs and their "macroscopic" photosensitive areas. This is the real challenge before us along with further electronics integration to be able to deal with at least on the order of 64 or more detection channels in parallel. In this way, it will become possible to carry out TD tomographic data acquisition in reasonable time without mechanical movement of detectors, which is currently the limiting factor to increase acquisition 
speed. This is the purpose of the work undertaken here and currently pursued in our laboratories.

\section{Conflict of Interests}

The authors declare that there is no conflict of interests regarding the publication of this paper.

\section{Acknowledgments}

Yves Bérubé-Lauzière acknowledges financial support from FRQ-NT (Projet de Recherche en Équipe), NSERC (Discovery Grant), and CFI (New Opportunities Fund) for this work. Yves Bérubé-Lauzière is member of the FRQ-S-funded Centre de Recherche du Centre Hospitalier Universitaire de Sherbrooke (CR-CHUS).

\section{References}

[1] L. Wang and $\mathrm{H}$. Wu, Biomedical Optics-Principles and Imaging, Wiley-Interscience, 2007.

[2] A. D. Klose, "Radiative transfer of luminescence light in biological tissue," in Light Scattering Reviews 4, Springer Praxis Books, chapter 6, pp. 293-345, Springer, Berlin, Germany, 2009.

[3] American National Standard for Safe Use of Lasers-ANSI Z136.1-2014, Laser Institute of America (LIA) and American National Standards Institute (ANSI), 2014.

[4] Safety of Laser Products Part 1: Equipment Classification and Requirements-IEC 60825-1:2014, Edition 3, International Electrotechnical Commission, Geneva, Switzerland, 2014.

[5] S. Prahl and S. Jacques, Optical Properties Spectra, 2015, http:// omlc.org/spectra/index.html.

[6] R. B. Buxton, Introduction to Functional Magnetic Resonance Imaging: Principles and Techniques, Cambridge University Press, Cambridge, UK, 2009.

[7] E. Ranyuk, R. Lebel, Y. Bérubé-Lauzière et al., " ${ }^{68} \mathrm{Ga} / \mathrm{DOTA}-$ and ${ }^{64} \mathrm{Cu} / \mathrm{NOTA}$-phthalocyanine conjugates as fluorescent/ PET bimodal imaging probes," Bioconjugate Chemistry, vol. 24, no. 9, pp. 1624-1633, 2013.

[8] M. L. James and S. S. Gambhir, "A molecular imaging primer: modalities, imaging agents, and applications," Physiological Reviews, vol. 92, no. 2, pp. 897-965, 2012.

[9] J. Wang, S. Jiang, Z. Li et al., "In vivo quantitative imaging of normal and cancerous breast tissue using broadband diffuse optical tomography," Medical Physics, vol. 37, no. 7, pp. 3715$3724,2010$.

[10] Q. Fang, J. Selb, S. A. Carp et al., "Combined optical and x-ray tomosynthesis breast imaging," Radiology, vol. 258, no. 1, pp. 89-97, 2011.

[11] M. L. Flexman, H. K. Kim, J. E. Gunther et al., "Optical biomarkers for breast cancer derived from dynamic diffuse optical tomography," Journal of Biomedical Optics, vol. 18, no. 9, Article ID 096012, 2013.

[12] R. Choe, S. D. Konecky, A. Corlu et al., "Differentiation of benign and malignant breast tumors by in-vivo threedimensional parallel-plate diffuse optical tomography," Journal of Biomedical Optics, vol. 14, no. 2, Article ID 024020, 2009.

[13] L. D. Montejo, J. Jia, H. K. Kim et al., "Computer-aided diagnosis of rheumatoid arthritis with optical tomography, part 2: image classification," Journal of Biomedical Optics, vol. 18, no. 7, Article ID 076002, 2013.
[14] D. Piao, K. E. Bartels, Z. Jiang et al., "Alternative transrectal prostate imaging: a diffuse optical tomography method," IEEE Journal on Selected Topics in Quantum Electronics, vol. 16, no. 4, pp. 715-729, 2010.

[15] K. K.-H. Wang and T. C. Zhu, "Reconstruction of in-vivo optical properties for human prostate using interstitial diffuse optical tomography," Optics Express, vol. 17, no. 14, pp. 11665-11672, 2009.

[16] A. P. Gibson, T. Austin, N. L. Everdell et al., “Three-dimensional whole-head optical tomography of passive motor evoked responses in the neonate," NeuroImage, vol. 30, no. 2, pp. 521$528,2006$.

[17] D. K. Joseph, T. J. Huppert, M. A. Franceschini, and D. A. Boas, "Diffuse optical tomography system to image brain activation with improved spatial resolution and validation with functional magnetic resonance imaging," Applied Optics, vol. 45, no. 31, pp. 8142-8151, 2006.

[18] A. T. Eggebrecht, S. L. Ferradal, A. Robichaux-Viehoever et al., "Mapping distributed brain function and networks with diffuse optical tomography," Nature Photonics, vol. 8, no. 6, pp. 448454, 2014.

[19] R. Weissleder and U. Mahmood, "Molecular imaging," Radiology, vol. 219, no. 2, pp. 316-333, 2001.

[20] T. F. Massoud and S. S. Gambhir, "Molecular imaging in living subjects: seeing fundamental biological processes in a new light," Genes and Development, vol. 17, no. 5, pp. 545-580, 2003.

[21] A. H. Hielscher, "Optical tomographic imaging of small animals," Current Opinion in Biotechnology, vol. 16, no. 1, pp. 79-88, 2005.

[22] V. Ntziachristos, "Fluorescence molecular imaging," Annual Review of Biomedical Engineering, vol. 8, pp. 1-33, 2006.

[23] F. Leblond, S. C. Davis, P. A. Valdés, and B. W. Pogue, "Preclinical whole-body fluorescence imaging: review of instruments, methods and applications," Journal of Photochemistry and Photobiology B: Biology, vol. 98, no. 1, pp. 77-94, 2010.

[24] H. Youn and K.-J. Hong, "In vivo noninvasive small animal molecular imaging," Osong Public Health and Research Perspectives, vol. 3, no. 1, pp. 48-59, 2012.

[25] G. Bouchard, G. Bouvette, H. Therriault, R. Bujold, C. Saucier, and B. Paquette, "Pre-irradiation of mouse mammary gland stimulates cancer cell migration and development of lung metastases," British Journal of Cancer, vol. 109, no. 7, pp. 18291838, 2013.

[26] E. Lapointe, J. Pichette, and Y. Bérubé-Lauzière, "A multi-view time-domain non-contact diffuse optical tomography scanner with dual wavelength detection for intrinsic and fluorescence small animal imaging," Review of Scientific Instruments, vol. 83, no. 6, Article ID 063703, 2012.

[27] S. L. Jacques, C. A. Alter, and S. A. Prahl, "Angular dependence of HeNe laser light scattering by human dermis," Lasers in the Life Sciences, vol. 1, no. 4, pp. 309-333, 1987.

[28] W. Cheong, S. Prahl, and A. Welch, "Optical properties of tissues in vitro," IEEE Journal of Quantum Electronics, vol. 12, no. 12 , pp. 2166-2185, 1990.

[29] S. K. Sharma and S. Banerjee, "Role of approximate phase functions in Monte Carlo simulation of light propagation in tissues," Journal of Optics A: Pure and Applied Optics, vol. 5, no. 3, pp. 294-302, 2003.

[30] A. D. Klose and E. W. Larsen, "Light transport in biological tissue based on the simplified spherical harmonics equations," Journal of Computational Physics, vol. 220, no. 1, pp. 441-470, 2006. 
[31] J. Bouza-Domínguez and Y. Bérubé-Lauzière, "Diffuse light propagation in biological media by a time-domain parabolic simplified spherical harmonics approximation with raydivergence effects," Applied Optics, vol. 49, no. 8, pp. 1414-1429, 2010.

[32] R. Berg, S. Andersson-Engels, and S. Svanberg, Time-Resolved Transillumination Imaging, vol. IS11, SPIE Press, 1993.

[33] J. Pichette, S. Boucher, G. B. Domínguez, and Y. BérubéLauzière, "Diffuse photon density wavefront speed as a contrast for tomographic imaging of heterogeneous diffusive media," Optics Letters, vol. 39, no. 7, pp. 2097-2100, 2014.

[34] J. Pichette, J. B. Domínguez, and Y. Bérubé-Lauzière, “Timedomain geometrical localization of point-like fluorescence inclusions in turbid media with early photon arrival times," Applied Optics, vol. 52, no. 24, pp. 5985-5999, 2013.

[35] F. Leblond, H. Dehghani, D. Kepshire, and B. W. Pogue, "Earlyphoton fluorescence tomography: spatial resolution improvements and noise stability considerations," Journal of the Optical Society of America A, vol. 26, no. 6, pp. 1444-1457, 2009.

[36] N. Valim, J. Brock, and M. Niedre, "Experimental measurement of time-dependant photon scatter for diffuse optical tomography," Journal of Biomedical Optics, vol. 15, no. 6, Article ID 065006, 2010.

[37] N. Valim, J. Brock, M. Leeser, and M. Niedre, "The effect of temporal impulse response on experimental reduction of photon scatter in time-resolved diffuse optical tomography," Physics in Medicine and Biology, vol. 58, no. 2, pp. 335-349, 2013.

[38] J. Bouza Domínguez and Y. Bérubé-Lauzière, "Diffuse optical tomographic imaging of biological media by time-dependent parabolic SPN equations: a two-dimensional study," Journal of Biomedical Optics, vol. 17, no. 8, Article ID 086012, 14 pages, 2012.

[39] W. Becker, A. Bergmann, and C. Biskup, "Multispectral fluorescence lifetime imaging by TCSPC," Microscopy Research and Technique, vol. 70, no. 5, pp. 403-409, 2007.

[40] C. Biskup, T. Zimmer, L. Kelbauskas et al., "Multi-dimensional fluorescence lifetime and FRET measurements," Microscopy Research and Technique, vol. 70, no. 5, pp. 442-451, 2007.

[41] W. Becker, The bh TCSPC Handbook, Becker \& Hickl GmbH, Berlin, Germany, 5th edition, 2012.

[42] V. Venugopal, J. Chen, M. Barroso, and X. Intes, "Quantitative tomographic imaging of intermolecular FRET in small animals," Biomedical Optics Express, vol. 3, no. 12, pp. 3161-3175, 2012.

[43] J. R. Lakowicz, Principles of Fluorescence Spectroscopy, Springer, New York, NY, USA, 3rd edition, 2006.

[44] G. Bodi and Y. Bérubé-Lauzière, "A new deconvolution technique for time-domain signals in diffuse optical tomography without a priori information," in Diffuse Optical Imaging II, vol. 7369 of Proceedings of SPIE, Munich, Germany, July 2009.

[45] M. Diop and K. St Lawrence, "Deconvolution method for recovering the photon time-of-flight distribution from timeresolved measurements," Optics Letters, vol. 37, no. 12, pp. 23582360, 2012.

[46] B. W. Pogue, T. O. McBride, U. L. Osterberg, and K. D. Paulsen, "Comparison of imaging geometries for diffuse optical tomography of tissue," Optics Express, vol. 4, no. 8, pp. 270-286, 1999.

[47] U. J. Netz, J. Beuthan, and A. H. Hielscher, "Multipixel system for gigahertz frequency-domain optical imaging of finger joints," Review of Scientific Instruments, vol. 79, no. 3, Article ID 034301, 2008.
[48] PerkinElmer's large product line of preclinical small animal optical imaging systems (IVIS R series, MaestroTM, and FMT 2500TM LX), PerkinElmer of Caliper Life Sciences, Xenogen Corp, Cambridge Research Instruments (CRi), and VisEn Medical, http://www.perkinelmer.com/.

[49] Biospace Lab, PhotonIMAGER ${ }^{\mathrm{Tw}}$, http://www.biospacelab.com/.

[50] N. Zarif Yussefian, M. Letendre-Janiaux, and Y. BérubéLauzière, "Continuous wave optical scanner for small animal optical molecular imaging," in Biomedical Optics Topical Meeting (OSA-BIOMED), BM3A-55, Optical Society of America, 2014.

[51] R. Lebel, N. Zarifyussefian, M. Letendre-Jauniaux et al., "Ultrahigh sensitivity detection of bimodal probes at ultra-low noise for combined fluorescence and positron emission tomography imaging," in Multimodal Biomedical Imaging VIII, vol. 8574 of Proceedings of SPIE, p. 7, SPIE BiOS-Photonics West, San Francisco, Calif, USA, February 2013.

[52] J. A. Guggenheim, H. R. A. Basevi, J. Frampton, I. B. Styles, and H. Dehghani, "Multi-modal molecular diffuse optical tomography system for small animal imaging," Measurement Science and Technology, vol. 24, no. 10, Article ID 105405, 2013.

[53] A. Koenig, A. Planat-Chrétien, K. Hassler et al., "Validation of an xct/fdot system on mice," ISRN Optics, vol. 2012, Article ID 735231, 13 pages, 2012.

[54] M. L. Flexman, F. Vlachos, H. K. Kim et al., "Monitoring early tumor response to drug therapy with diffuse optical tomography," Journal of Biomedical Optics, vol. 17, no. 1, Article ID 016014, 2012.

[55] Y. Lin, W. C. Barber, J. S. Iwanczyk, W. Roeck, O. Nalcioglu, and G. Gulsen, "Quantitative fluorescence tomography using a combined tri-modality FT/DOT/XCT system," Optics Express, vol. 18, no. 8, pp. 7835-7850, 2010.

[56] J. M. Masciotti, J. Lee, M. Stewart, and A. H. Hielscher, "Instrumentation for simultaneous magnetic resonance and optical tomographic imaging of the rodent brain," in Multimodal Biomedical Imaging IV, vol. 7171 of Proceedings of SPIE, p. 16, San Jose, Calif, USA, February 2009.

[57] L. Hervé, A. Koenig, A. Da Silva et al., "Noncontact fluorescence diffuse optical tomography of heterogeneous media," Applied Optics, vol. 46, no. 22, pp. 4896-4906, 2007.

[58] N. Deliolanis, T. Lasser, D. Hyde, A. Soubret, J. Ripoll, and V. Ntziachristos, "Free-space fluorescence molecular tomography utilizing $360^{\circ}$ geometry projections," Optics Letters, vol. 32, no. 4, pp. 382-384, 2007.

[59] R. B. Schulz, G. Echner, H. Ruhle et al., "Development of a fully rotational non-contact fluorescence tomographer for small animals," in Proceedings of the IEEE Nuclear Science Symposium Conference Record, vol. 4, pp. 2391-2393, Fajardo, Puerto Rico, October 2005.

[60] V. Ntziachristos, C.-H. Tung, C. Bremer, and R. Weissleder, "Fluorescence molecular tomography resolves protease activity in vivo," Nature Medicine, vol. 8, no. 7, pp. 757-760, 2002.

[61] J. H. Lee, H. K. Kim, C. Chandhanayingyong, F. Y.-I. Lee, and A. H. Hielscher, "Non-contact small animal fluorescence imaging system for simultaneous multi-directional angular-dependent data acquisition," Biomedical Optics Express, vol. 5, no. 7, pp. 2301-2316, 2014.

[62] Y. Lin, M. T. Ghijsen, H. Gao, N. Liu, O. Nalcioglu, and G. Gulsen, "A photo-multiplier tube-based hybrid MRI and frequency domain fluorescence tomography system for small animal imaging," Physics in Medicine and Biology, vol. 56, no. 15, pp. 4731-4747, 2011. 
[63] C. D. Darne, Y. Lu, I.-C. Tan et al., "A compact frequencydomain photon migration system for integration into commercial hybrid small animal imaging scanners for fluorescence tomography," Physics in Medicine and Biology, vol. 57, no. 24, pp. 8135-8152, 2012.

[64] J. C. Rasmussen, A. Joshi, T. Pan, T. Wareing, J. McGhee, and E. M. Sevick-Muraca, "Radiative transport in fluorescenceenhanced frequency domain photon migration," Medical Physics, vol. 33, no. 12, pp. 4685-4700, 2006.

[65] Y. Lu, C. D. Darne, I. C. Tan et al., "Experimental comparison of continuous-wave and frequency-domain fluorescence tomography in a commercial multi-modal scanner," IEEE Transactions on Medical Imaging, vol. 34, no. 6, pp. 1197-1211, 2015.

[66] B. Montcel and P. Poulet, "An instrument for small-animal imaging using time-resolved diffuse and fluorescence optical methods," Nuclear Instruments and Methods in Physics Research Section A: Accelerators, Spectrometers, Detectors and Associated Equipment, vol. 569, no. 2, pp. 551-556, 2006.

[67] D. Kepshire, N. Mincu, M. Hutchins et al., "A microcomputed tomography guided fluorescence tomography system for small animal molecular imaging," Review of Scientific Instruments, vol. 80, no. 4, Article ID 043701, 2009.

[68] M. J. Niedre, G. M. Turner, and V. Ntziachristos, "Time-resolved imaging of optical coefficients through murine chest cavities," Journal of Biomedical Optics, vol. 11, no. 6, Article ID 064017, 2006.

[69] M. Niedre and V. Ntziachristos, "Comparison of fluorescence tomographic imaging in mice with early-arriving and quasicontinuous-wave photons," Optics Letters, vol. 35, no. 3, pp. 369371, 2010.

[70] R. E. Nothdurft, S. V. Patwardhan, W. Akers, Y. Ye, S. Achilefu, and J. P. Culver, "In vivo fluorescence lifetime tomography," Journal of Biomedical Optics, vol. 14, no. 2, Article ID 024004, 2009.

[71] A. T. N. Kumar, S. B. Raymond, A. K. Dunn, B. J. Bacskai, and D. A. Boas, "A time domain fluorescence tomography system for small animal imaging," IEEE Transactions on Medical Imaging, vol. 27, no. 8, pp. 1152-1163, 2008.

[72] D. J. Cuccia, F. Bevilacqua, A. J. Durkin, and B. J. Tromberg, "Modulated imaging: quantitative analysis and tomography of turbid media in the spatial-frequency domain," Optics Letters, vol. 30, no. 11, pp. 1354-1356, 2005.

[73] V. Venugopal, J. Chen, F. Lesage, and X. Intes, "Full-field timeresolved fluorescence tomography of small animals," Optics Letters, vol. 35, no. 19, pp. 3189-3191, 2010.

[74] V. Venugopal, J. Chen, and X. Intes, "Development of an optical imaging platform for functional imaging of small animals using wide-field excitation," Biomedical Optics Express, vol. 1, no. 1, pp. 143-156, 2010.

[75] S. R. Arridge, “Optical tomography in medical imaging," Inverse Problems, vol. 15, no. 2, pp. R41-R93, 1999.

[76] W. Becker, Advanced Time-Correlated Single Photon Counting Techniques, Springer, New York, NY, USA, 1st edition, 2005.

[77] J. Pichette, Imagerie de fluorescence et intrinsèque de milieux diffusants par temps d'arrivée des premiers photons [Ph.D. thesis], Université de Sherbrooke, 2014.

[78] Y. Bérubé-Lauzière and V. Robichaud, “Time-resolved fluorescence measurements for diffuse optical tomography using ultrafast time-correlated single photon counting," in Advanced Photon Counting Techniques, W. Becker, Ed., vol. 6372 of Proceedings of SPIE, Boston, Mass, USA, October 2006.
[79] A. Gulinatti, I. Rech, P. Maccagnani, M. Ghioni, and S. Cova, "Large-area avalanche diodes for picosecond time-correlated photon counting," in Proceedings of the 35th European SolidState Device Research Conference (ESSDERC '05), pp. 355-358, Grenoble, France, September 2005.

[80] S. Cova, M. Ghioni, F. Zappa, I. Rech, and A. Gulinatti, "A view on progress of silicon single-photon avalanche diodes and quenching circuits," in Proceedings of the Advanced Photon Counting Techniques, vol. 6372 of Proceedings of SPIE, The International Society for Optical Engineering, Boston, Mass, USA, October 2006.

[81] C. Cammi, F. Panzeri, A. Gulinatti, I. Rech, and M. Ghioni, "Custom single-photon avalanche diode with integrated frontend for parallel photon timing applications," Review of Scientific Instruments, vol. 83, no. 3, Article ID 033104, 8 pages, 2012.

[82] M. Crotti, I. Rech, and M. Ghioni, "Four channel, 40 ps resolution, fully integrated time-to-amplitude converter for time-resolved photon counting," IEEE Journal of Solid-State Circuits, vol. 47, no. 3, pp. 699-708, 2012.

[83] S. Antonioli, L. Miari, A. Cuccato, M. Crotti, I. Rech, and M. Ghioni, "8-channel acquisition system for time-correlated single-photon counting," Review of Scientific Instruments, vol. 84, no. 6, Article ID 064705, 2013.

[84] R. Yao, Q. Pian, and X. Intes, "Wide-field fluorescence molecular tomography with compressive sensing based preconditioning," Biomedical Optics Express, vol. 6, no. 12, pp. 4887-4898, 2015.

[85] A. Goetzberger, B. Mcdonald, R. H. Haitz, and R. M. Scarlett, "Avalanche effects in silicon $p-n$ junctions. II. Structurally perfect junctions," Journal of Applied Physics, vol. 34, no. 6, pp. 1591-1600, 1963.

[86] R. H. Haitz, "Model for the electrical behavior of a microplasma," Journal of Applied Physics, vol. 35, no. 5, pp. 1370-1376, 1964.

[87] R. H. Haitz, "Mechanisms contributing to the noise pulse rate of avalanche diodes," Journal of Applied Physics, vol. 36, no. 10, pp. 3123-3131, 1965.

[88] M. Ghioni, S. Cova, A. Lacaita, and G. Ripamonti, "New silicon epitaxial avalanche diode for single-photon timing at room temperature," Electronics Letters, vol. 24, no. 24, pp. 1476-1477, 1988.

[89] S. Cova, A. Lacaita, M. Ghioni, G. Ripamonti, and T. A. Louis, "20-ps timing resolution with single-photon avalanche diodes," Review of Scientific Instruments, vol. 60, no. 6, pp. 1104-1110, 1989.

[90] A. Lacaita, M. Ghioni, and S. Cova, "Double epitaxy improves single-photon avalanche diode performance," Electronics Letters, vol. 25, no. 13, pp. 841-843, 1989.

[91] A. Lacaita, S. Cova, M. Ghioni, and F. Zappa, "Single-photon avalanche diode with ultrafast pulse response free from slow tails," IEEE Electron Device Letters, vol. 14, no. 7, pp. 360-362, 1993.

[92] A. Spinelli, M. A. Ghioni, S. D. Cova, and L. M. Davis, "Avalanche detector with ultraclean response for time-resolved photon counting," IEEE Journal of Quantum Electronics, vol. 34, no. 5, pp. 817-821, 1998.

[93] M. Ghioni, A. Gulinatti, I. Rech, P. Maccagnani, and S. Cova, "Large-area low-jitter silicon single photon avalanche diodes," in Quantum Sensing and Nanophotonic Devices V, vol. 6900 of Proceedings of SPIE, International Society for Optical Engineering, San Jose, Calif, USA, February 2008. 
[94] C. Cammi, A. Gulinatti, I. Rech, F. Panzeri, and M. Ghioni, "SPAD array module for multi-dimensional photon timing applications," Journal of Modern Optics, vol. 59, no. 2, pp. 131139, 2012.

[95] C. Veerappan, J. Richardson, R. Walker et al., "A 160x128 singlephoton image sensor with on-pixel 55ps 10b time-to-digital converter," in Proceedings of the IEEE International Solid-State Circuits Conference Digest of Technical Papers (ISSCC '11), pp. 312-314, San Francisco, Calif, USA, February 2011.

[96] D. Tyndall, B. R. Rae, D. D. Li et al., "A high-throughput timeresolved mini-silicon photomultiplier with embedded fluorescence lifetime estimation in $0.13 \mu \mathrm{m}$ cmos," IEEE Transactions on Biomedical Circuits and Systems, vol. 6, no. 6, pp. 562-570, 2012.

[97] F. Villa, B. Markovic, S. Bellisai et al., "SPAD smart pixel for time-of-flight and time-correlated single-photon counting measurements," IEEE Photonics Journal, vol. 4, no. 3, pp. 795$804,2012$.

[98] D. Stoppa, F. Borghetti, J. Richardson et al., “A 32×32-pixel array with in-pixel photon counting and arrival time measurement in the analog domain," in Proceedings of the 35th European SolidState Circuits Conference (ESSCIRC '09), pp. 204-207, Athens, Greece, September 2009.

[99] C. Niclass, C. Favi, T. Kluter, M. Gersbach, and E. Charbon, "A $128 \times 128$ single-photon image sensor with column-level 10bit time-to-digital converter array," IEEE Journal of Solid-State Circuits, vol. 43, no. 12, pp. 2977-2989, 2008.

[100] M. Ghioni, A. Gulinatti, P. Maccagnani, I. Rech, and S. Cova, "Planar silicon SPADs with 200- $\mu \mathrm{m}$ diameter and 35-ps photon timing resolution," in Proceedings of the Single Photon Avalanche Detectors and Superconducting Detectors II, vol. 6372 of Proceedings of SPIE, Advanced Photon Counting Techniques, The International Society for Optical Engineering, Boston, Mass, USA, October 2006.

[101] A. Gulinatti, I. Rech, F. Panzeri et al., "New silicon SPAD technology for enhanced red-sensitivity, high-resolution timing and system integration," Journal of Modern Optics, vol. 59, no. 17, pp. 1489-1499, 2012.

[102] S. Cova, M. Ghioni, A. Lacaita, C. Samori, and F. Zappa, "Avalanche photodiodes and quenching circuits for singlephoton detection," Applied Optics, vol. 35, no. 12, pp. 1956-1976, 1996.

[103] A. Gallivanoni, I. Rech, and M. Ghioni, "Progress in quenching circuits for single photon avalanche diodes," IEEE Transactions on Nuclear Science, vol. 57, no. 6, pp. 3815-3826, 2010.

[104] A. Spinelli and A. L. Lacaita, "Physics and numerical simulation of single photon avalanche diodes," IEEE Transactions on Electron Devices, vol. 44, no. 11, pp. 1931-1943, 1997.

[105] A. Gulinatti, P. Maccagnani, I. Rech, M. Ghioni, and S. Cova, "35 ps time resolution at room temperature with large area single photon avalanche diodes," Electronics Letters, vol. 41, no. 5, pp. 272-274, 2005.

[106] M. Crotti, I. Rech, A. Gulinatti, and M. Ghioni, "Avalanche current read-out circuit for low-jitter parallel photon timing," Electronics Letters, vol. 49, no. 16, pp. 1017-1018, 2013.

[107] M. Crotti, I. Rech, and M. Ghioni, "Monolithic time-toamplitude converter for TCSPC applications with 45 ps time resolution," in Proceedings of the 7th Conference on Ph.D. Research in Microelectronics and Electronics (PRIME '11), pp. 2124, Trento, Italy, July 2011.

[108] M. Kanoun, Y. Bérubé-Lauzière, and R. Fontaine, "High precision time-to-amplitude converter for diffuse optical tomography applications," in Proceedings of the IEEE International
Conference on Design and Technology of Integrated Systems in Nanoscale Era (DTIS '08), pp. 1-4, IEEE, Tozeur, Tunisia, March 2008.

[109] B. Markovic, S. Tisa, F. A. Villa, A. Tosi, and F. Zappa, "A high-linearity, 17 ps precision time-to-digital converter based on a single-stage Vernier delay loop fine interpolation," IEEE Transactions on Circuits and Systems. I. Regular Papers, vol. 60, no. 3, pp. 557-569, 2013.

[110] J. Richardson, R. Walker, L. Grant et al., "A $32 \times 3250 \mathrm{ps}$ resolution 10 bit time to digital converter array in 130nm CMOS for time correlated imaging," in Proceedings of the IEEE Custom Integrated Circuits Conference (CICC '09), pp. 77-80, San Jose, Calif, USA, September 2009.

[111] A. Cuccato, S. Antonioli, M. Crotti et al., "Complete and compact 32-channel system for time-correlated single-photon counting measurements," IEEE Photonics Journal, vol. 5, no. 5, 14 pages, 2013.

[112] M. Crotti, I. Rech, and M. Ghioni, "Note: fully integrated time-to-amplitude converter in Si-Ge technology," Review of Scientific Instruments, vol. 81, no. 10, Article ID 106103, 2010.

[113] C. Cottini, E. Gatti, and V. Svelto, "A new method for analog to digital conversion," Nuclear Instruments and Methods, vol. 24, pp. 241-242, 1963.

[114] S. Antonioli, M. Crotti, A. Cuccato, I. Rech, and M. Ghioni, "Time-correlated single-photon counting system based on a monolithic time-to-amplitude converter," Journal of Modern Optics, vol. 59, no. 17, pp. 1512-1524, 2012.

[115] F.-J. Luo, Y.-S. Yin, S.-Q. Liang, and M.-L. Gao, "Current switch driver and current source designs for high-speed currentsteering DAC," in Proceedings of the 2nd International Conference on Anti-counterfeiting, Security and Identification (ASID '08), pp. 364-367, IEEE, Guiyang, China, August 2008.

[116] I. Benamrane and Y. Savaria, "Design techniques for high speed current steering DACs," in Proceedings of the IEEE North-East Workshop on Circuits and Systems (NEWCAS '07), pp. 14851488, Montreal, Canada, August 2007.

[117] Y. Bérubé-Lauzière, V. Robichaud, and É. Lapointe, “Timeresolved non-contact fluorescence diffuse optical tomography measurements with ultra-fast time-correlated single photon counting avalanche photodiodes," in Diffuse Optical Imaging of Tissue, vol. 6629 of Proceedings of SPIE, p. 8, Munich, Germany, July 2007.

[118] L. Spinelli, D. Contini, R. Cubeddu et al., "Brain functional imaging at small source-detector distances based on fast-gated single-photon avalanche diodes," in Photonic Therapeutics and Diagnostics V, vol. 7161 of Proceedings of SPIE, p. 7, San Jose, Calif, USA, January 2009.

[119] A. Tosi, A. Dalla Mora, F. Zappa et al., "Fast-gated singlephoton counting technique widens dynamic range and speeds up acquisition time in time-resolved measurements," Optics Express, vol. 19, no. 11, pp. 10735-10746, 2011.

[120] M. Mazurenka, A. Jelzow, H. Wabnitz et al., "Non-contact timeresolved diffuse reflectance imaging at null source-detector separation," Optics Express, vol. 20, no. 1, pp. 283-290, 2012.

[121] Y. Mu, N. Valim, and M. Niedre, "Evaluation of a fast singlephoton avalanche photodiode for measurement of early transmitted photons through diffusive media," Optics Letters, vol. 38, no. 12, pp. 2098-2100, 2013. 

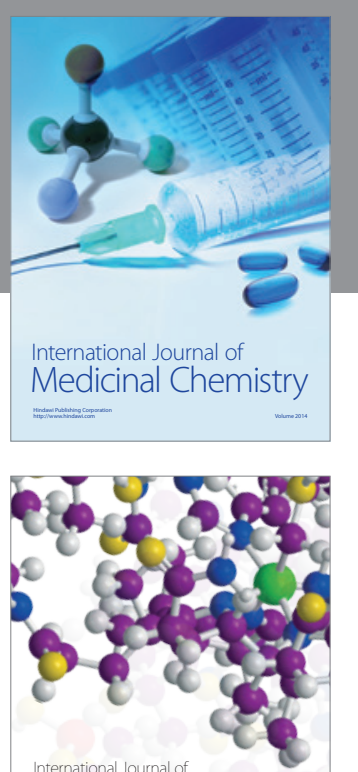

Carbohydrate Chemistry

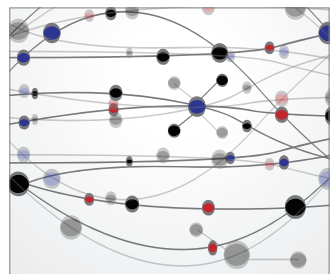

The Scientific World Journal
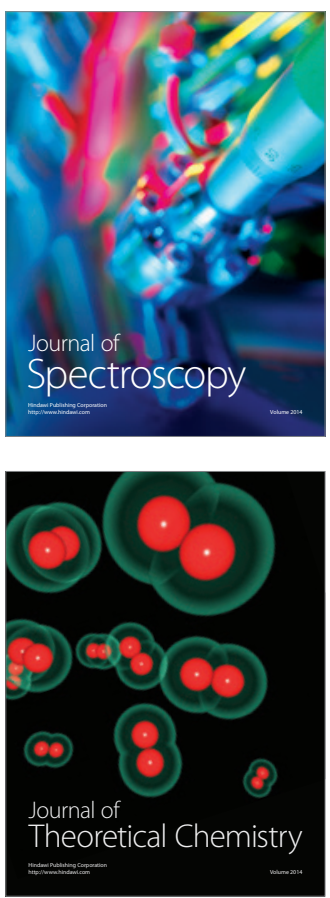
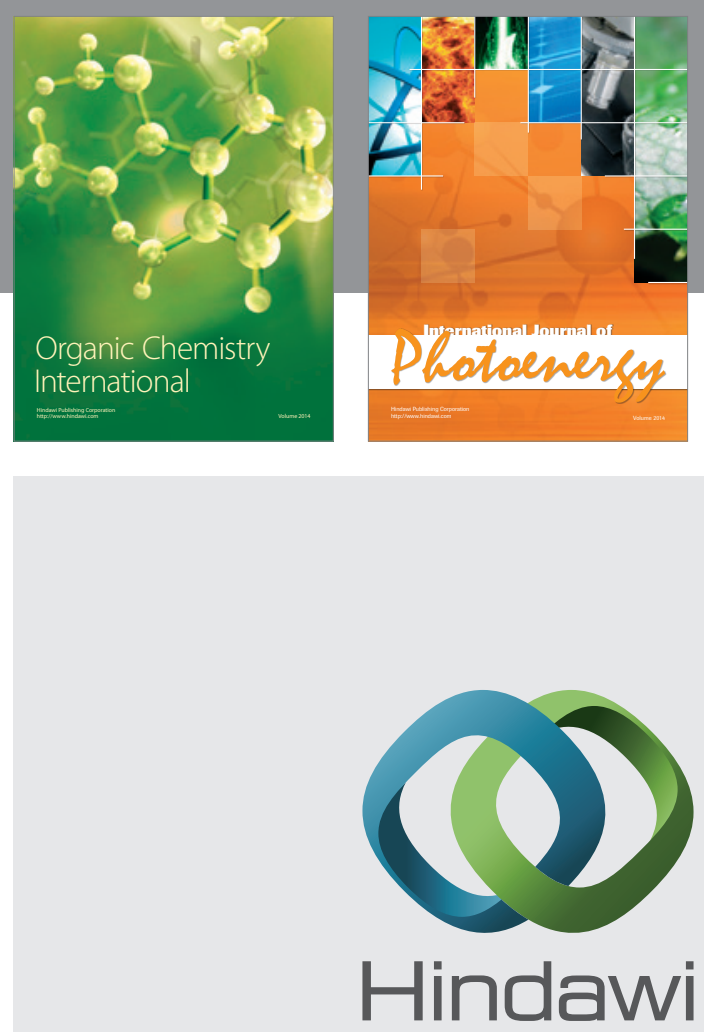

Submit your manuscripts at

http://www.hindawi.com

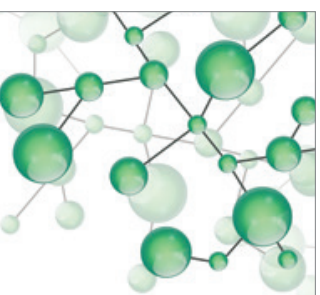

International Journal of

Inorganic Chemistry

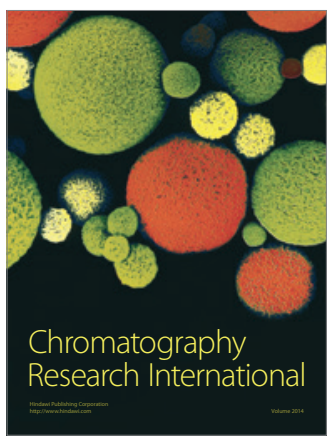

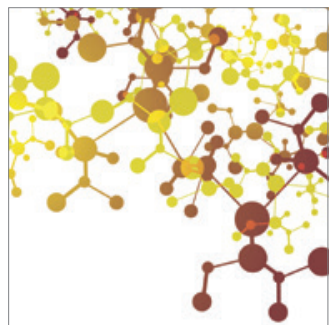

Applied Chemistry
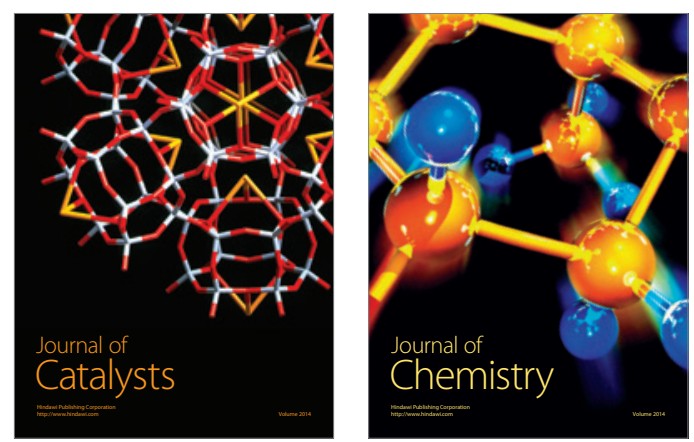
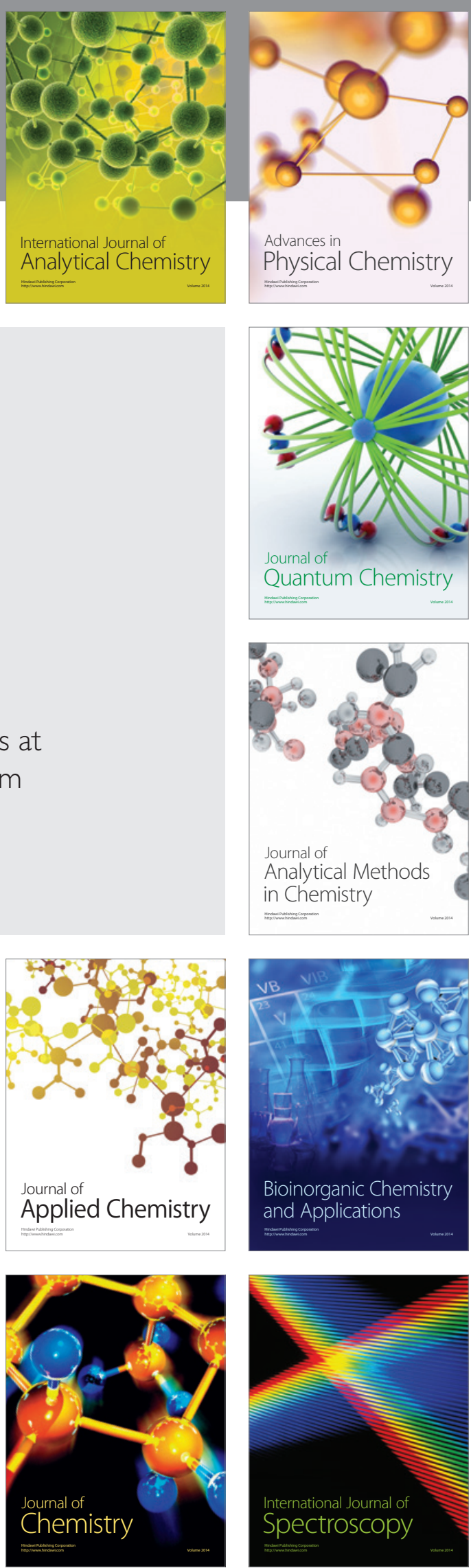